Carbonel Frédéric

Doctorant en Histoire (Université de Rouen)

\title{
Origines et développement de l'Institut havrais de sociologie économique et de psychologie des peuples de l'hiver 1937 aux années 1980.
}

\section{Introduction}

«Il y a dans la psychologie des peuples un fond de permanence qui se retrouve toujours » (André Siegfried, L'âme des peuples, 1950).

L'Institut Havrais de Sociologie économique et de Psychologie des Peuples a été fondé deux ans avant la Seconde guerre mondiale au moment où s'ouvrait une période de dislocation du Front Populaire à partir de la fin 1937. Alors que la République française conservait encore son immense empire colonial, la crise politique internationale et militaire annonçait l'effondrement probable de la III $^{\mathrm{e}}$ République devant l'éventualité d'une nouvelle guerre mondiale. Dans un premier temps, nous verrons les rapports entre la naissance de la psychologie des peuples et l'essor de l'idéologie républicaine au tournant du siècle, époque de la «République coloniale ${ }^{1} »$ où s'élabora une «psychologie collective » qui va annoncer la science politique du $\mathrm{XX}^{\mathrm{e}}$ siècle. Dans un second temps, nous nous attarderons sur la figure emblèmatique du politologue havrais André Siegfried (1875-1959) et sur son rôle pour la fondation d'une «psychologie des peuples» en tant que discipline «scientifique" française à part entière. Enfin, nous verrons l'implantation et le rayonnement de cette dernière à partir de la création d'un Institut Havrais au cours de l'hiver 1937 jusqu'aux années 1980. Tout en montrant ses développement, puis ses atermoiements, dans un nouveau contexte de guerre froide et de décolonisation, nous insisterons sur l'action d'un autre protestant havrais qui eut une fonction de premier plan dans cet essor: le philosophe Abel Miroglio. Par ailleurs, nous tenterons de montrer que contrairement à une certaine idée reçue la psychologie des peuples n'a pas été un obstacle à l'émergence des sciences dites sociales mais au contraire on peut dire qu'elle a reçu un véritable succès à la fois local et national, (voire international) $^{2}$. En effet, elle obtint de nombreux soutiens au Havre et en France mais aussi à l'étranger surtout au cours des années 1950-1960. Pour l'ensemble, nous essayerons d'appréhender quelles en furent les raisons économiques et sociologiques mais aussi historiques et culturelles.

\section{I-LA NAISSANCE DE LA PSYCHOLOGIE DES PEUPLES ET LE RENFOR- CEMENT DE L'IDEOLOGIE REPUBLICAINE AU TOURNANT DU XIXE- XXE SIECLE (ANNEES 1890-ANNEES 1910)}


Alfred Fouillée (1838-1912) joua un rôle de premier plan auprès des élites françaises au cours des années 1890-1910. Il fut aussi membre associé de la Société de Psychologie physiologique dès sa fondation en $1886^{3}$. Fouillée établit une continuité à travers ses écrits entre la «psychologie collective » et la «psychologie des Peuples » initiée d'un côté par Gabriel Tarde, auteur des Lois de l'imitation (1890) et de La logique sociale (1895), et de l'autre par Gustave Le Bon, auteur de la Psychologie des foules (1895) et des Lois psychologiques de l'évolution des peuples $(1894)^{4}$.

\section{1-Alfred Fouillée, de la «psychologie collective» à la «psychologie des peuples ${ }^{5}$}

Époux de Mme Bruno, l'auteur du célèbre best seller Le Tour de France par deux enfants paru en $1877^{6}$, Fouillée produisit de nombreux articles et ouvrages sur les droits nationaux dont en 1878, L'idée moderne du droit en Allemagne, en Angleterre et en France, en 1895, Tempéraments et caractères selon les individus, les sexes et les races et en 1910, La démocratie politique et sociale en France. Fouillée avait pour projet l'élaboration d'une synthèse républicaine qui ferait consensus à la fin du $\mathrm{XIX}^{\mathrm{e}}$ siècle et au début du $\mathrm{XX}^{\mathrm{e}}$ siècle. Il débuta comme maître de conférence à l'E.N.S. en 1872 puis fut élu à l'Académie des Sciences Morales et Politiques en 1894. Ses livres furent publiés dans 16 langues différentes. Quels furent ses rapports avec l'émergence d'une «psychologie des Peuples »?

Fouillée s'opposait vigoureusement au déterminisme racial de l'allemand Otto Ammon, théoricien de l'anthroposociologie ${ }^{7}$, car pour lui il n'y avait pas de « races » au sens propre mais des «peuples» auxquels il accolait pourtant des souscatégories : «types » et «sous races ». Si Fouillée semble s'être dressé fermement contre les idées de Vacher de Lapouge, « socialiste raciste » qui se rapprochait alors $\mathrm{du}$ darwiniste social allemand Ammon (les théories de Vacher de Lapouge et Ammon furent associées par la suite et appelées «Lois » Lapouge-Ammon ${ }^{8}$ ), dans ses travaux on pouvait tout de même retrouver des thèses issues de l'anthroposociologie. Cependant, Fouillée s'inscrivait comme un précurseur du solidarisme : il s'affiliait à la tradition des philosophes républicains extrêmement critiques vis à vis des théories racistes issues du «darwinisme social ». En ce sens, Fouillée voulait mettre davantage en évidence les «caractères » de la nation (tout comme celui du génie national issu des principes de la Révolution Française de 1789) qui étaient alors perçus comme tout à fait opposés aux «caractères » allemands du pangermanisme et de l'anthropologie raciale. Pour Fouillée, la «psychologie des peuples » prouvait son utilité par ses objectifs initiaux : assimiler et assumer le génie national et les origines de la «nation » à travers le système éducatif par exemple. C'est pourquoi il écrivit un Enseignement au point de vue national en 1891. La décennie suivante Fouillée fut remarqué pour ses travaux sur la Psychologie du peuple français (1898) et son Esquisse psychologique des peuples européens (1903). Le «caractère national » était bien, selon Fouillée, « intimement lié au tempérament qui lui-même est lié à la constitution héréditaire et aux traits ethniques, non moins qu'au milieu physique ${ }^{9} »$. Il est souvent considéré à ce titre comme un savant précurseur de la «Psychologie des peuples » mais plutôt dans une 
version française de ce schéma qui a pu inspirer en partie les fondateurs de l'Institut Havrais plus d'une génération après lui.

\section{2-«Quotient intellectuel », « race » et « psychologie des peuples »}

À la fin du $\mathrm{XIX}^{\mathrm{e}}$ siècle la psychologie devint discipline universitaire avec Théodule Ribot, Pierre Janet et Alfred Binet (fondateur de L'Année psychologique en 1895). Les premiers psychologues trouvèrent d'autres collaborateurs, avec une vision «large » de leur discipline originelle, comme Alfred Fouillée mais aussi Émile Boutmy, le fondateur de l'École libre des Sciences Politiques en 1872. A cette époque, l'intellect, l'imaginaire, le normal et le pathologique étaient dans le même temps l'objet de plus grandes considérations dans un contexte où ces nouveaux instruments disciplinaires, ancêtres des futures «sciences humaines », avaient pour objectif principal d'arriver à une meilleure connaissance des individus mais aussi à celle des «masses ${ }^{10}$. Or, ces nouvelles disciplines entretenaient des relations privilégiées avec d'autres sciences (jugées plus anciennes: la physiologie, l'anthropologie, la craniologie, l'anthropométrie, la physiognomonie ; mais aussi plus modernes : la criminologie, la psychologie expérimentale et différentielle, et la psychiatrie). Ces dernières représentaient une part non négligeable des articles de l'Année Psychologique comme de l'Année sociologique dans une époque politique et culturelle où la recherche des lois de l'hérédité (puis de la génétique) régissant les comportements humains supposait une étude des différences individuelles et des inégalités. Pour cela, l'anglais Francis Galton (1822-1911), cousin de Charles Darwin, s'était attaché à renforcer les études d'anthropologie culturelle et de psychologie différentielle tout en aboutissant à une pensée eugénique (invention du terme en 1883). Dans une perspective différente mais non éloignée, la psychologie expérimentale française s'attacha aussi à classer les individus en fonction d'indices mesurables. Elle permit l'institution, par exemple, des fameux tests mentaux BinetSimon reconnus par l'Instruction Publique dans l'objectif prioritaire d'une meilleure instruction pour tous les enfants français $(1905,1908$ et 1911) mais aussi pour mieux détecter les « handicaps » et les « inadaptations ${ }^{11}$. Des tests du quotient intellectuel (qui s'inspiraient des tests français en les élargissant) eurent au même moment un fort écho au sein de la psychologie américaine mais avec des considérations bien opposées puisqu'ils permettaient de classer les «catégories » de la population dans les années 1910-1920 en fonction des «communautés» et des «besoins»: L'intelligence des enfants chinois à San Francisco ; les Caractères psychologiques des Nègres des Etats du Sud; la Psychologie différentielle des Juifs; Le domaine de la psychologie des races; l'État actuel de la question des différences entre races; l'Intelligence des Blancs et des Nègres. Ces «enquêtes » eurent un tel succès aux États-Unis que lors du recrutement des cadres de l'armée américaine en 1917 on crut s'apercevoir des faibles scores des soldats noirs à la différence des WASP (White Anglo Saxon Protestants) ${ }^{12}$. Plutôt détachée de tels intérêts pseudo-scientifiques ${ }^{13}$, la psychologie française développa au tournant du $\mathrm{XIX}^{\mathrm{e}}-\mathrm{XX}^{\mathrm{e}}$ siècles plusieurs approches de la «psychologie » : celle des « foules ${ }^{14}$ mais aussi celle des «individus» (notamment avec Pierre Janet ${ }^{15}$ ) et des «peuples ». Ces approches multiples et complexes répondaient aux préoccupations républicaines du temps avec ces interro- 
gations centrales sur le fonctionnement des individus mais aussi sur celui de la «nation ${ }^{16}$. Ce fut au coeur de ces sujets, entre autres, que s'inscrivirent particulièrement les travaux d'André Siegfried, l'un des principaux promoteurs de l'Institut Havrais de psychologie des peuples ${ }^{17}$.

\section{3-André Siegfried: le parcours d'un "grand havrais» ou une figure emblématique de la « Psychologie des peuples »?}

André Siegfried fut une figure charismatique de la $\mathrm{III}^{\mathrm{e}}$ puis de la $\mathrm{IV}^{\mathrm{e}}$ République $^{18}$. Représentant éminent de la science politique française dans les années qui suivirent la Libération, il fut sous la $\mathrm{IV}^{\mathrm{e}}$ République un proche du président René Coty qui avait bien connu son père. Professeur au collège de France, il fut nommé membre de l'Institut puis président du conseil d'administration de l'École libre des Sciences Politiques. Il y poursuivit ses cours pendant la seconde guerre mondiale tout comme au Collège de France. Il cumula ces fonctions avec celle de membre de l'Académie des Sciences Morales et Politiques ainsi que celle de président de l'Association Française de Science Politique. Sous le régime de Vichy, il aurait continué à professer des cours sur les « races » tout en restant bien silencieux sur la législation antisémite organisée par Pétain : des lois de «discriminations légales » furent mise en place sous le Régime de Vichy dès juillet-octobre $1940^{19}$. André Siegfried avait été désigné pour être membre du Conseil National de Vichy, poste qu'il refusa à la différence de "petits déjeûners », sans doute circonstanciés (?), à l'Institut dépendant de l'ambassade d'Allemagne en janvier-février $1941^{20}$.

Avant la guerre, après des études de Droit et de Lettres, André Siegfried avait étudié pendant une année à l'École libre des Sciences Politiques. Il y avait suivi l'enseignement de Boutmy, le fondateur de l'École. Par la suite, toute sa carrière professionnelle d'enseignant et d'administrateur fut attachée à la vie de cette institution où il avait été nommé professeur titulaire de chaire dès 1910. Une vingtaine d'années auparavant, au cours de ses jeunes années, il avait pu assister aux discussions des membres (ou anciens membres) de la Société de psychologie physiologique, notamment dans des soirées parisiennes tenues par Madame Marillier en compagnie de Lucien Herr alors très intéressé par la Völkerpsychologie de Wundt (à la fin des années 1880 et au début des années 1890 selon l'historien Charles Andler un des premiers à dénoncer les effets conjugués du racisme «néo-darwinien » et du pangermanisme ${ }^{21}$ ). Ceci incita certainement très tôt André Siegfried à étudier la «psychophysiologie » qui influença la plupart de ses travaux sur les Français mais aussi sa vision des «étrangers $»^{22}$.

Siegfried fut élu en octobre 1944 à l'Académie Française puis devint chroniqueur au Figaro poursuivant des articles qu'il avait publiés au Temps, d'octobre 1941 au 18 novembre 1942. Ce dernier quotidien avait été maréchaliste et administré par Joseph Barthelemy, un ancien professeur de l'École libre de Sciences Politiques, nommé ministre de la Justice par Pétain ${ }^{23}$. De nombreux chercheurs on put noter dans son oeuvre les influences de Barrès, et sans doute de manière indirecte celles de 
Jules Soury (1842-1915), titulaire de la première chaire d'histoire des doctrines psychologiques à l'École Pratique des Hautes Études en $1881^{24}$. Jules Soury était un proche de Barrès, qu'il convertit dans ses cours à ses centres d'intérêt (il fut aussi maurrassien). Soury avait fait une synthèse paradoxale entre Gobineau et Renan dans son enseignement «psycho-physiologique» devenu officiel à l'E.P.H.E. Ainsi, plusieurs historiens, après avoir attentivement lu plusieurs écrits d'André Siegfried y ont vu les relents d'un antisémitisme « modéré » qui pouvait être mis en rapport avec le livre de chevet préféré d'André Siegfried ( selon ses propres propos ) : le Roman de l'énergie nationale, la trilogie du très nationaliste Maurice Barrès ${ }^{25}$. Or, sous la III $^{\mathrm{e}}$ République, à la Libération puis tout au long de la $\mathrm{IV}^{\mathrm{e}}$ République et jusqu'aux débuts de la $\mathrm{V}^{\mathrm{e}}$ République André Siegfried demeura un pilier de l'establishment culturel français incontesté voire encensé. Par ailleurs, son attitude sous le régime de Vichy, alors qu'il était un expert et un savant reconnu, n'avait-elle pas été en contradiction avec celle de son père au moment de l'affaire Dreyfus ${ }^{26}$ ?

La thèse de doctorat d'André Siegfried parue en 1913, Tableau politique de la France de l'Ouest, contribua fortement à sa réputation ${ }^{27}$. Il y donnait une explication pluraliste des phénomènes politiques: «D’une façon générale, je me suis volontairement méfié de l'explication unique, de la clef qui prétend ouvrir toutes les serrures (...). Je crois au contraire que, dans cette matière complexe, il faut presque toujours recourir à un grand nombre de causes, dont aucune ne serait à elle seule suffisante, mais dont le faisceau apporte de précieuse clarté ». C'est ce qu'il appellera par la suite une « analyse combinatoire ». Néanmoins, on peut aujourd'hui constater le fort penchant d'André Siegfried pour un certain «déterminisme géographique et ethnique» qui était sensiblement partagé par de nombreux intellectuels français de son époque. Ses premières publications et son enseignement pourraient ainsi le situer dans le droit fil d'Hippolyte Taine, de Gustave Lebon, de Georges Vacher de Lapouge voire d'Otto Ammon, à la différence de Fouillée plus détaché $^{28}$. Il faut ici rappeler que la formation intellectuelle d' A. Siegfried se situait à la veille de la première guerre mondiale, période où «l'anthropo-géographie » allemande était à son âge d'or. Or, en réaction devant les préoccupations du théoricien allemand Friedrich Ratzel, l'élite française se forgeait contre celle-ci avec un double regard à la fois de répulsion mais aussi de fascination depuis la première guerre franco-prusienne ${ }^{29}$. Pour André Siegfried, les notions de «tempérament » et de «caractère » demeuraient les concepts premiers d'explication historique d'une société donnée. C'étaient ces derniers qui permettaient de comprendre la «complexe personnalité de la nation » de même que les «tempéraments politiques régionaux », les «provinces », les « régions », les « cantons » et les «pays ». Dans cette optique, l'étude de la « race bretonne», autrement dit des habitants natifs de Bretagne qui pratiquaient encore les «dialectes celtiques », pouvait facilement devenir synonyme celle du « caractère breton ${ }^{30}$. Pour André Siegfried, il pouvait y avoir « des régions politiques comme il y a des régions géologiques ou économiques, et des climats politiques comme il y a des climats naturels ${ }^{31} \gg$. 
Document $\mathbf{n}^{\circ}$ 1: Extrait du «passé de la psychologie des peuples» d'A. Miroglio, alors directeur honoraire du centre de Psychologie des peuples havrais, en $1971^{[32]}$.

Jetons un regard, avant de terminer ce chapitre sur ce que nous apporte l'Allemagne pendant cette dernière période qui précède immédiatement notre actualité. Il y a essentiellement l'Anthropogéographie de Ratzel et la Völkerpsychologie de Wundt. Ratzel est généralement reconnu, du fait de son Anthropogeographie, comme le fondateur de la géographie politique entendue au sens de la détermination du mode d'existence et de l'histoire des peuples par la pression toute-puissante des facteurs géographiques. Bien sûr, il a été un initiateur ; mais la juste critique des excès de cette philosophie selon laquelle la position (die Lage) et l'étendue (der Raum) d'un état le prédestinent à telle ou telle expansion, on la trouve dans toute l'œuvre de Vidal de La Blache et de ses continuateurs français.

\section{4-Les influences du mouvement colonial, la « sociologie économique » et ses conséquences sociales et culturelles au Havre des années 1880 aux années 1940.}

A notre avis, la première thèse de lettres d'André Siegfried, réalisée en 1904, nous fait mieux comprendre ce personnage, les préoccupations de son milieu d'origine mais aussi indirectement la création de l'Institut Havrais : Edward Gibbon Wakefield et sa doctrine de la colonisation systématique ${ }^{33}$. Le père d'André Siegfried joua un rôle prépondérant pour éveiller ses centres d'intérêts et pour son orientation future tout comme Jacques Siegfried, son oncle banquier ${ }^{34}$. Ainsi une typologie en trois grandes thématiques de l'œuvre scientifique d'André Siegfried, premier président d'honneur de l'Institut Havrais, illustrerait parfaitement les approches possibles de la «Psychologie des Peuples »: l'étude de la vie politique française et du caractère politique national ; l'étude du monde anglo-saxon ; et enfin l'étude des problèmes du monde contemporain, vécu par A. Siegfried, y incluant ses aspects coloniaux ${ }^{35}$. L'ensemble semblait bien marqué par la géographie de Paul Vidal de la Blache (1845-1918), qui avait fait le compte-rendu de sa thèse en $1914^{36}$, ainsi que par l'école historio-géographique française du début du $\mathrm{XX}^{\mathrm{e}}$ siècle (qui tout en se démarquant de l'anthropogéographie allemande en intégrait paradoxalement plusieurs éléments) ${ }^{37}$. L'intérêt pour les pays anglo-saxons et leurs particularités y était singulièrement perceptible notamment dans Le Canada, les deux races publié en 1906 (Siegfried pouvait y trouver des «modèles » expressément révélateurs de sa pensée mais aussi de ses visions géographiques). Les «tempéraments nationaux »y formaient des principes explicatifs généraux mais aussi «ethniques » pour la compréhension du peuple canadien ou américain par exemple. Nous pouvons retrouver une grille de lecture similaire dans American Comes of Ages. A French Analysis, publié avec un grand succès en $1927^{38}$. Siegfried y abordait la question de l'antisémitisme américain protestant issu, d'après lui, d'un réflexe d'autodéfense (en rapport à la peur des invasions ainsi qu'aux nouvelles vagues d'immigration). Il y faisait non seulement écho aux quotas pour les immigrants en vertu des lois de 1921 mais aussi à celles de Johnson-Reed votées en 
1924. Toute sa réflexion pouvait renvoyer implicitement aux interrogations françaises du moment sur le «tempérament national» et la «naturalisation ${ }^{39}$. De même, dans Vue générale de la Méditerranée, publié en 1943, André Siegfried n'hésita pas à faire une classification des « races » alors que le décret Crémieux avait été abrogé le 7 octobre 1940 supprimant aux juifs d'Algérie leurs droits de citoyenneté. Mais, après la seconde guerre mondiale, son ouvrage de 1950, L'âme des peuples, laissera à plusieurs historiens un certain malaise, comme l'indiqua Pierre Favre en $1989^{40}$. L'historien israélien Zeev Sternhell jugea carrément cette étude «raciste » voire «antisémite " ${ }^{41}$, accusation que Gérard Noiriel put réitérer concernant le premier best-seller d'André Siegfried sur les États-Unis aujourd'hui ${ }^{42}$. La traduction littérale du titre anglais semblerait d'ailleurs plus explicite et plus spécifiquement liée à l'idée d'un « inconscient collectif » ainsi qu'à la «psychologie des peuples »: L'Amérique du fond des âges. Une analyse française ${ }^{43}$. André Siegfried insistait toujours sur les déterminismes « ethniques » et «psychologiques » qui imposaient, selon lui, un cadre conceptuel de compréhension des nations et des peuples. Ainsi, sa lecture privilégiait l'étude des «tempéraments » comme continuité et permanence des populations au même titre qu'il pouvait le faire pour les « Normands » ou les « Bretons », voire les «traditions » politiques et culturelles paysannes enracinées localement. L'homogénéité territoriale et « ethnique » constituait bien pour A. Siegfried une constance politique à travers les époques et les générations. Cette position analytique caractéristique fut bien plus tard reprise par les «cercles » de la rue Saint-Guillaume, notamment autour de François Goguel. En revanche, elle se démarqua bien plus nettement du déterminisme physiologique lié au Volkisch, (la conception dite «allemande du peuple »). La tradition politique française prit alors plus nettement pour fil conducteur celle du Volkgeist (le génie national). Nous pouvons penser que cette opposition entre ces deux conceptions de la «Psychologie des Peuples », « allemande » ou «française », avait été curieusement explicitée bien des années auparavant par l'historien Ernest Lavisse qui écrivait dès 1887 : «L'Allemagne a cette vocation : revendiquer pour elle tout ce qui est germanique, exalter le germanisme, développer dans l'univers la puissance germanique. Quelle est la nôtre? Il n'y a pas de doute que nous avons charge de représenter la cause de l'humanité ${ }^{44} »$. Déjà de la «psychologie des peuples »?

Le père d'André Siegfried avait été le principal artisan de l'École libre des Sciences Politiques, avec Émile Boutmy, protestant comme lui ${ }^{45}$. Boutmy sera l'auteur en 1901 d'une Psychologie du peuple anglais. Il avait participé à l'ouverture du Musée Social en 1895 à l'époque où Dick May fondait l'École des Hautes Etudes Sociales dans le sud de Paris ${ }^{46}$. Les activités du père d'André ${ }^{47}$, Jules Siegfried (1837-1922), pouvaient expliquer en amont et a posteriori les balbutiements de cette vogue de «psychologie des peuples ». Jules Siegfried, en effet, maire républicain modéré du Havre de 1878 à 1885, avait joué un rôle de premier plan dans l'émergence et le soutien massif à la propagande coloniale. Le Havre, second port français, à une semaine en paquebot de Dakar et des Etats-Unis, était alors surnommé la New-York française ${ }^{48}$. La Porte Océane importait des produits chers (coton, café, cacao et bois exotiques) des colonies d'A.O.F., d'A.E.F., d'Indochine et de Madagascar. Le premier port haut-normand possédait depuis 1907 une École 
Pratique coloniale. Protestant d'origine alsacienne, fils d'un modeste industriel du textile, Jules Siegfried, le père d'André, était devenu millionnaire à 29 ans car il fut l'un des premiers à comprendre que la guerre de sécession aux Etats-Unis (18611865) priverait la France de l'approvisionnement en coton. Il eut alors l'idée d'aller en Inde pour importer lui-même cette matière première nécessaire au textile normand. Dès cette époque, il apparut comme un fervent admirateur de cet «esprit d'entreprise », regardé comme typique des pays anglo-saxons et protestants, comme les Etats-Unis et la Grande-Bretagne ${ }^{49}$. Jules Siegfried était admiratif de leurs hommes politiques (notamment d'Abraham Lincoln plus favorable au protectionnisme que les états du sud des Etats-Unis, aspirant à plus de libre échange donc non abolitionnistes ${ }^{50}$ ). Jules Siegfried fut nommé ministre du Commerce et de l'Industrie dans le premier cabinet Ribot puis ministre du Commerce et des Colonies dans un second cabinet en janvier 1893. Il fut le créateur des Habitations à Bon Marché, participait à la Société pour la lutte contre les taudis. Il avait aussi fondé des clubs de loisirs dans les cités ouvrières. Il était à la Ligue de l'enseignement et il fut aussi membre fondateur du Comité national républicain du commerce et de l'industrie avec Waldeck-Rousseau (1846-1904) et le jeune Raymond Poincaré (1860-1834) en avril 1897. Il appartenait enfin à la Société d'aide de protection des colons depuis 1898. Jules Siegfried, le père d'André, fut ainsi le principal dirigeant du Groupe colonial du Sénat (fondé la même année avec de grands noms de la politique coloniale opportuniste comme le républicain social, opportuniste et protestant de progrès Richard Waddington (1838-1913), président de la Chambre de commerce de Rouen). Ce groupe devait en lien avec les chambres de commerce locales garantir les principaux intérêts économiques liés à l'expansion coloniale française $^{51}$. En mai 1901 Jules Siegfried, à la fois patriote et fervent dreyfusard, s'inscrivit avec les « républicains du centre gauche » dans l'Alliance Républicaine démocratique qui regroupait les républicains laïques, modérés et libéraux (dont un nombre important proche des milieux d'affaires).

Ce fut à cette époque qu'apparut la sociologie économique (c'est à dire l'étude des faits économiques éclairés par la sociologie). Au cours des décennies 1890-1920, des sociologues élaborèrent des méthodes d' « analyse combinée » de psychologie, de sociologie et d'économie: Max Weber, fondateur de l'association allemande de sociologie en 1908 ; Schumpeter, auteur de la Théorie de l'évolution économique en 1912, et dans une moindre mesure (en ce qui concerne spécialement la sociologie économique) le français Durkheim ${ }^{52}$. D'ailleurs ce dernier dénonça les approches pseudo-«culturelles » de plusieurs «savants » français dont le docteur Letourneau (1831-1902), titulaire de la chaire d'histoire des civilisations à l'Ecole d'anthropologie de Paris depuis 1885. Letourneau dans le contexte de la diffusion des idées du «darwinisme social » faisait de La sociologie d'après l'ethnographie (1884) l'axe de La guerre dans les diverses races humaines (1895) mais aussi de La psychologie ethnique (1901) ${ }^{53}$.

Au début des années 1930, Albert Charles, président de la commission des colonies à la Chambre de commerce du Havre, l'un des futurs présidents de l'Institut Havrais, négociant en bois coloniaux, dirigeant de l'Institut colonial du Havre et du 
Comité pour l'Indochine française, avait été un des premiers à exposer sa vision d'une exposition coloniale internationale. Son vœu fut exaucé lors de l'exposition coloniale de 1931 où l'« ethnocentrisme » européen pouvait triompher avec son parc zoologique et ses milliers d'indigènes figurants ${ }^{54}$. De même en 1940, on trouva, malgré l'actualité dramatique, à l'exposition du Grand Palais à Paris un stand intitulé «Le Havre, grand port colonial » qui suscita la grande curiosité des visiteurs.

\section{5-André Siegfried, premier président d'honneur de l'Institut Havrais.}

Juste avant, puis après la seconde guerre mondiale, André Siegfried donna son appui personnel à la fondation d'un Institut havrais, ville à laquelle il était particulièrement attaché. Dans son premier numéro de mai 1946 la revue de l'Institut, publiée sous plusieurs patronages dont le sien, mais aussi celui d'autres personnalités comme le psychiatre et professeur Laignel-Lavastine (1875-1953), ancien président de la Société Médico-Psychologique (1939) et ancien membre de la Ligue d'hygiène et de prophylaxie mentale (fondée en 1920), le comité de rédaction écrivait : «Mais, demandera-t-on, pourquoi cette nouveauté nous arrive-t-elle du Havre ?». Le même comité précisait : «Elle témoigne de notre souci d'humanisme et de psychologie des peuples qui est également fort bien situé au Havre, ville dont la population est d'origine très composite, ville de grands voyageurs, ville où les contacts avec l'étranger sont quotidiens ». Le philosophe protestant Abel Miroglio, principal animateur de l'Institut Havrais, n'a jamais manqué dans ses travaux de souligner l'étroite filiation intellectuelle qui avait pu exister avec son maître André Siegfried pour cette entreprise : « en tous cas, c'est du fait des très abondantes et très fines touches de psychologie des peuples, éparses dans cette œuvre si vaste, qu'a été éveillée en nous la vocation de redonner un élan à cette science délicate, si mal acclimatée en France ${ }^{55}$ ». Il revendiquait toujours plusieurs années après cette continuité : «il nous a semblé que dans la ville d'André Siegfried, ce merveilleux observateur qui, sans prononcer l'expression de psychologie des peuples, était passionnément intéressé par la réalité que ces mots recouvrent, nous ferions bien d'élire cette discipline à laquelle dans ses ouvrages il a fourni un excellent apport ${ }^{56}{ }$.

Il faut ici souligner qu'à la fin des années trente, un autre «savant » essaya de donner une assise scientifique à l'approche "psycho-géographique » de la «personnalité collective ». C'est Georges Hardy qui publia en 1939 La Géographie psychologique $^{57}$, un vrai manifeste théorique. Georges Hardy (de son vrai nom Gabriel Giroud) établissait un inventaire quasi exhaustif des variables d'observation des comportements collectifs. Concernant ces explications il écrivait : "elles sont loin ... de s'exercer toutes en même temps et dans tous les cas ... Le plus souvent, nous avons à faire à des influences combinées ». À l'aube d'un second conflit mondial, ces approches pouvaient constituer une orientation de recherche et une avancée décisive pour l'étude de la politique et de la géographie. La personnalité collective des populations était particulièrement bien soulignée par Georges Hardy mais c'est la notion d' "âme collective » qu'il pensait particulièrement bien mettre en évidence... 
De son côté André Siegfried avait aussi mis l'accent sur le tempérament politique national ainsi que les dimensions variables de la «personnalité des peuples »: «il dissèque les nations et en démonte la mécanique » notait Paul Claval ${ }^{58}$. En effet, A. Siegfried mettait à jour les structures fondamentales des milieux géographiques ainsi que les facteurs qui pesaient sur la vie des états et des régions car elles pouvaient influencer les orientations longues des populations. Quant à Miroglio, il faisait apparaître que pour Siegfried la géopolitique était «engagée dans un complexe spirituel beaucoup plus vaste » qui amenait à la «psychologie des peuples » ${ }^{59}$. La réflexion générale du premier président d'honneur de l'Institut Havrais sur les caractéristiques de l'environnement géographique, les pesanteurs historiques et leurs héritages culturels serviraient de fil conducteur à ses recherches comme l'évoqua Miroglio à plusieurs reprises, notamment lors de trois conférences scientifiques aux Journées d'étude du Havre sur les méthodes de la psychologie des peuples en 1948, au Congrès philosophique international d'Amsterdam la même année et au Congrès de l'Association française pour l'avancement des sciences de Caen en 1955.

Document $n^{\circ} 2$ : André Siegfried vu par l'historien britannique Théodore Zeldin ${ }^{60}$.

Protestant et fils d'un millionnaire parti de rien, à la manière américaine, il avait voyagé pendant sa jeunesse dans le monde entier, parlait avec « un accent presque étranger » et portait des costumes de tweed comme un globetrotter sorti tout droit d'un roman de Jules Verne. À quatre reprises (19021910) il se présenta aux élections législatives. Battu, comme Tocqueville, il se lança dans l'étude des mystères d'une vie politique à laquelle il ne parvenait pas à s'adapter. Dans un ouvrage très ingénieux, intitulé Tableau politique de la France de l'Ouest (1913), il s'est servi des connaissances locales détaillées qu'il avait acquises au cours de ses campagnes électorales pour démontrer que les divisions politiques pouvaient être étroitement rattachées à des «tempéraments » régionaux. "Tout comme il existe des tempéraments individuels, écrivait-il, il existe des tempéraments provinciaux et nationaux ». Dans ce livre, il tentait de «traduire en termes conscients l'inconscient profond de ces psychologies ».

Document $\mathbf{n}^{\circ} 3$ : La fondation de l'Institut Havrais d'après A. Miroglio en $1971^{61}$.

On voudra bien nous permettre d'acheminer les lecteurs vers l'actualité en partant de l'époque immédiatement consécutive à la première guerre mondiale. C'est d'ailleurs à cette époque que la psychologie des peuples a failli être lancée, sous le nom d' « éthologie collective »; un beau programme a été conçu et l'initiateur était d'envergure, et excellemment placé pour décider des hommes à se mettre au travail : il s'agissait d'Henri Berr, qui avait esquissé un grand programme se reliant évidemment à sa pensée maîtresse, celle de la 
«synthèse historique ». Un des mérites de Henri Berr, a été de définir la notion de psychologie régionale et de ne pas se borner à la théorie : neuf ouvrages ont été édités sous sa direction sur les régions de la France.

En 1939, juste au seuil de la seconde guerre mondiale, c'est un véritable manifeste en faveur de la psychologie des peuples qui paraît avec La géographie psychologique de Georges Hardy. Le moment était bien mauvais ; en 1940, la psychologie des peuples ne pouvait être envisagée qu'à la lumière de la guerre, c'est à dire sous l'influence de la pire des conseillères.

C'est au cours de l'hiver 1937-1938, donc peu de temps avant la parution du livre de Hardy, que l'auteur de ce livre fondait au Havre, avec des concours locaux, l'Institut havrais de Sociologie économique et de Psychologie des peuples auquel immédiatement André Siegfried, très attaché au Havre, sa ville natale, accordait sa confiance en acceptant la présidence d'honneur. Plus d'un quart de siècle s'est écoulé depuis, et cet Institut a donné une franche priorité à la psychologie des peuples, terrain de ses succès décisifs, ce qu'est venu sanctionner le nouveau titre qu'il a reçu depuis son officialisation, survenue en 1960, et qui en a fait un Institut de l'Université de Caen, puis, à partir de 1965, de l'Université de Rouen. Il s'appelle désormais le Centre de Recherches et d'Études de Psychologie des peuples et de Sociologie économique. Depuis mai 1946 cet Institut du Havre publie, avec une parfaite régularité, de trimestre en trimestre, une Revue de psychologie des peuples qui est sans analogue dans le monde de langue française et qui aujourd'hui rayonne en une soixantaine de pays étrangers ; on la trouve dans la plupart des bibliothèques des universités françaises et dans un très grand nombre de bibliothèques étrangères du vieux et du nouveau continent.

\section{II-L'INSTITUT HAVRAIS : L'IMPACT DE SA FONDATION, SA METHODE ET SES OBJECTIFS DE 1937 AUX ANNEES 1970.}

À la veille puis après la seconde guerre mondiale la «psychologie des peuples » avait l'ambition de s'élever comme une authentique discipline universitaire avec ses champs de recherche et ses spécialités propres.

\section{1-L'Institut Havrais, sa création et ses premiers objectifs : le rôle primordial des coloniaux ${ }^{62}$.}

Ses initiateurs envisageaient l'Institut comme une «école scientifique » à part entière selon E. Callot, qui fut un temps président de l'Institut ${ }^{63}$. L'implantation havraise favorisait les échanges grâce à sa vocation portuaire océanique marquée par une forte activité liée au commerce : malgré la montée des mouvements nationalistes au sein des pays colonisés, juste après la Libération, l' «Union Française » permit plusieurs années après la seconde guerre mondiale de maintenir un empire colonial français. Néanmoins deux vagues de décolonisation marquées par deux guerres 
coloniales en Indochine (1945-1954) et en Algérie (1954-1962) se succédèrent. Dans ce nouveau contexte où de nouvelles relations internationales apparurent, l'Institut Havrais dut se positionner face à l'anthropologie culturaliste américaine, nouvelle « idéologie » devenue à la mode ${ }^{64}$. «L'école de psychologie des peuples » bien implantée au Havre se voulait une discipline originale mais non isolée. On peut aussi noter le rôle primordial des coloniaux français dans le fonctionnement de l'Institut sous la «République coloniale » dès ses origines.

En décembre 1937 avec l'aide et l'appui de concours locaux, d'entrepreneurs et d'élus, Abel Miroglio fondait l'Institut Havrais en tant que société scientifique privée avec un statut d'association. L'association bénéficia de l'action des coloniaux et de leurs sociabilités. Le 13 mars 1936, l'année qui précèda sa fondation, le gouverneur honoraire des colonies avait bien suscité l'éclosion d'associations procoloniales. Il proposait ainsi au sous-préfet de la Seine-Inférieure le patronage d'un Comité de propagande et d'action coloniale. La Société de Géographie commerciale du Havre ${ }^{65}$ tandis que les sections havraises et fécampoises de la Ligue maritime et coloniale vulgarisaient au même moment auprès des citoyens la «sociologie économique » et la «psychologie des peuples ». La sociabilité des élites havraises et particulièrement celle des coloniaux favorisa largement l'essor de l'Institut apportant dons financiers et soutiens moraux. L'Institut Havrais prétendait prolonger les activités de la Société de géographie commerciale qui s'effacera définitivement en 1948 après avoir amplement profité des apports de la nouvelle «géographie économique ».

Parmi les premiers mécènes de l'Institut se trouvaient des coloniaux qui avaient tout intérêt à ce type d'entreprise. Les hautes personnalités du négoce havrais étaient bien présentes dans le conseil d'administration et sa direction : Rodolphe Rufenacht (la Compagnie cotonnière, ancienne maison Ernest Siegfried, la Compagnie Fernand Lehoux avec ses succursales à Douala et Abidjan et la Société commerciale interocéanique avec ses comptoirs à Madagascar et en Côte d'Ivoire) ; Étienne Amphoux (la Maison Hauser) ; Louis Hubert (la Compagnie générale des cafés) ; Georges Vachoux (la Société franco-coloniale d'Importation de café et des denrées coloniales) ; Albert Charles (la première maison européenne de bois exotiques) ; Augustin Normand (la Compagnie franco-coloniale des riz). Le 18 novembre 1937, Georges Raverat, président de la chambre de commerce locale et de l'Union Congolaise, dirigeant de plusieurs compagnies commerciales (les sociétés commerciales de navigation et de banque, le commerce de l'ivoire et du caoutchouc) convoqua les premières personnalités qui allaient faire partie du premier comité d'organisation de l'Institut. C'est dans son bureau que naquit l'association. Le colonel Vachoux, son ami, fut choisi comme président. Les solidarités d'affaires favorisaient cette curiosité culturelle qui semblait défaillante à l'École supérieure de commerce du Havre alors que la propagande coloniale accroissait son contrôle social sur la ville portuaire. Le réseau se trouvait donc renforcé par des positions communes des administrateurs à la fois dans les assurances, la presse, l'armement (l'importation de nickel de Nouvelle Calédonie entre autres), les docks et magasins mais aussi la Banque de France et la Caisse de liquidation des affaires en marchandises. Ces centres d'intérêts furent une des raisons majeures du 
développement d'un courant favorable à un lieu de réflexion sur la «psychologie des peuples ». L'ethnologie, tout comme la «psychologie des peuples » puis «l'ethnopsychologie », semblaient alors étroitement liées au phénomène colonial ${ }^{66}$.

En 1925, à Paris, Lévy-Brühl avait créé l'Institut d'Ethnographie tandis que la Société des Africanistes prenait essor à la même époque au Musée d'Ethnographie du Trocadéro fondé en 1878, remplacé par le Musée de l'Homme en 1937. En 1931 c'était du Havre que sur le Saint-Firmin embarqua Michel Leiris pour la mission Dakar-Djibouti. La création de l'Institut Havrais en 1937 pouvait accompagner un certain «humanitarisme colonial et bienveillant» y compris encouragé sous le gouvernement du Front Populaire. De même, la curiosité ethnographique marquée par la «mission civilisatrice » et «l'éducation des races» pouvait largement s'accorder aux préoccupations commerciales ${ }^{67}$.

$\mathrm{Au}$ sein de l'Institut de nombreux coloniaux protestants, provenant de familles du coton, mais aussi des familles de la bourgeoisie catholique locale. En juillet 1937, Rodolphe Rufenacht et Miroglio s'assurèrent le soutien des parlementaires de la région ${ }^{68}$. Dans un document de travail soumis aux parlementaires locaux et offert au sous-préfet le 20 juillet 1937, Miroglio écrivait : «dans une ville qui possède une Bourse d'une exceptionnelle importance et où se traitent des affaires considérables, la sociologie économique est une science qui doit être tout naturellement élue. Si elle est traitée à la française, avec un souci de compréhension humaine, il apparait qu'elle est difficilement dissociable d'une psychologie des peuples, science plus jeune encore, mais riche d'avenir ». Pour ce dernier le soutien des réseaux d'affaires était bien source d'optimisme: "plusieurs personnalités du haut commerce havrais disposent de relations très étendues en de nombreux pays et ont assez d'envergure pour faire une utile propagande dans les universités étrangères au cours de leurs propres tournées d'affaires ». Miroglio pouvait déclarer lors de la séance inaugurale du 7 janvier 1938: «on ne peut traiter de sociologie économique en ignorant les colonies; or, nous avons au Havre des sociétés vouées à l'étude des problèmes coloniaux. Nous avons aussi une société de géographie commerciale ${ }^{69} »$. Le 15 mars 1948, l'Institut élargit ainsi son réseau aux correspondants de la Société de géographie : «la connexion avec la sociologie économique et la psychologie des peuples est salutaire » expliquaient les négociants intéressés. Dès la Libération l'Institut se fonda comme un élément à part entière du dispositif idéologique colonial qui louait alors directement les vertus de l'Union Française ${ }^{70}$.

Dans son «Que-sais-je? » sur la Psychologie des peuples, Miroglio revendiquait de nombreux héritages dont ceux d'Henri Berr, Max Weber, Roger Bastide et André Siegfried $^{71}$. Il est vrai qu'A. Siegfried avait appartenu en 1936 au « centre d'études des problèmes humains », une annexe du Centre d'études économiques de l'École Polytechnique (un cercle qui compta jusqu'à 1200 membres en 1937 et qui succédait pour certaines de ses activités à la Société de Biotypologie fondée en 1932 dont le but était, par exemple, d'organiser l'orientation et la sélection professionnelle des adultes). Le Centre d'études économiques avait été fondé en 1931, face à la crise économique, par Jean Coutrot (1895-1941), ancien élève de l'École libre de Sciences Politique, alors responsable des relations pour le monde du travail dans le 
gouvernement du Front Populaire. En outre, le «centre d'études des problèmes humains » avait regroupé de nombreux spécialistes, intellectuels, syndicalistes, technocrates et industriels formés en sciences politiques, en droit et sociologie mais aussi en psychiatrie et psychologie comme Aldous Huxley (1894-1963), l'auteur du Brave New World en 1932 (Le meilleur des mondes) ou le psychologue Henri Wallon (1879-1962), professeur au Collège de France depuis 1937, ainsi que l'auteur en 1935 de L'homme cet inconnu, Alexis Carrel (1873-1944) ${ }^{72}$. Il trouva une prolongation «dévoyée » sous Vichy à travers la Fondation française pour l'étude des problèmes humains (transformée à la Libération en I.N.E.D. sous l'impulsion d'Alfred Sauvy). La Fondation française pour l'étude des problèmes humains avait été elle-même créée par l'eugéniste Alexis Carrel le 17 novembre 1941, avec l'appui financier, et non sans intérêt, de l'Institut Rockefeller, mais aussi avec la collaboration de nombreux intellectuels, savants et experts français ${ }^{73}$. Toujours, à la suite des initiatives de Coutrot s'était organisé sur Paris un Institut de psychologie appliquée avec des commissions spécialisées qui fonctionna de 1936 à $1941^{74}$. À la veille de la Seconde guerre mondiale, l'Institut Havrais pouvait largement s'en inspirer d'autant que l'intérêt pour ses travaux pouvait être partagé par les réseaux coloniaux haut-normands.

\section{2-L'action du protestant Abel Miroglio, principal initiateur de l'Institut Havrais $^{75}$.}

Miroglio (7 février 1895 -1978) était le dernier de 5 enfants issus d'une vieille famille genevoise et italienne. Protestant, citoyen français et suisse, descendant des comtes de Miroglio de Moncesto (comtes du Saint-Empire Romain Germanique), Miroglio avait un père officier d'administration. Il avait fait ses études au lycée de Cherbourg puis en khagne à Lyon. Après la première guerre mondiale il poursuivit son parcours d'étudiant à l'E.N.S. puis au lycée de Metz. Il devint agrégé de philosophie après avoir hésité longtemps avec l'agrégation d'allemand. En 1928, il dirigea l'Ecole Internationale de Genève et enseigna à la Faculté des Lettres de Nancy. En 1931, il quitta Nancy pour Bordeaux puis obtint une mutation au Havre en 1936. Très influencé par Bergson, dont il fut l'ami, ainsi que par la sociologie, il devint membre de la Fédération des Associations Chrétiennes d'étudiants qui rassemblait alors catholiques, protestants et orthodoxes. Ancien élève aux langues $\mathrm{O}$ (à l'E.N.S.), il conserva un grand intérêt pour la culture russe et les pays de l'Est. De même pour l'Allemagne et ses relations avec la France, depuis qu'il avait obtenu une licence en géographie. Il fut aussi passablement influencé par la Völkerpsychologie de Wundt (1832-1920), que nous avons évoquée plus haut, initiateur de la psychologie expérimentale et auteur d'une monumentale Psychologie des peuples (une étude des «lois» de développement de la langue, des mythes et des mœurs) publiée en 10 tomes entre 1900 et 1920. Wundt était passé de la psychologie expérimentale à l'étude des «psychismes collectifs ». La Wölkerpsychologie voulait succéder à la Völkercharakterologie, jugée trop générale pour aboutir à une vraie «psychologie de la culture » axée sur les « sciences morales ${ }^{76} »$.

En 1963, Miroglio succèda à Jean-Paul Sartre au Lycée François I ${ }^{\text {er }}$ du Havre. Il portait alors un grand intérêt pour les identités urbaines ainsi qu'à la «géographie 
psychologique » grâce à son ami alias Georges Hardy, dont nous avons parlé plus haut, qui l'orienta très tôt dans cette direction. Miroglio pouvait ainsi réaliser son grand projet: fonder un Institut en vue d'un développement universitaire havrais dans la principale ville ouverte et «cosmopolite» de la Haute-Normandie. D'abord association privée aux multiples appuis dont la Mairie, la Chambre de Commerce, le Port autonome et le Rectorat, l'Institut Havrais prit son essor surtout après 1946 à la faveur d'un contexte propice et précis que nous avons en partie décrit : milieu du négoce, Institut Colonial, École Pratique coloniale, Société de Géographie économique. La première ambition de Miroglio fut d'accumuler une documentation portant sur l'Afrique, profitant du «prisme» exceptionnellement favorable des coloniaux français dans ce continent. Déjà en 1937, selon les archives de l'Institut, environ 30 universités avaient apporté leur caution au projet.

\section{3-Les activités de l'Institut et son rayonnement des années 1950 aux années $1960^{77}$.}

Au cours des années 1950-1960, «l'école française de psychologie des peuples » développa ses travaux parallèlement aux travaux «culturalistes » de l'anthropologue américaine Ruth Benedict, correspondante de l'Institut Havrais de 1946 à 1958, et de l'ethnologue Margaret Mead. Mais, l'association resta très élitaire : «pour être membre actif il faut être présenté par deux membres actifs et agréé par le Conseil d'administration par un vote à bulletin secret » précisaient les statuts. Alors qu'au départ l'Institut Havrais était plutôt hostile à l'anthropologie culturelle de type américain, ces membres allaient devoir opérer quelques reconversions puisque l'Institut recruta rapidement plus d'une centaine de nouveaux membres. Pendant la seconde guerre mondiale l'association avait poursuivi ses activités de façon intermittente, elle devint plus directement après 1946 un foyer de la recherche française centrée sur la «psychologie des peuples». Ainsi des années 1950 aux années 1960 des subventions régulières du C.N.R.S. lui furent attribuées. L'Institut adhéra dans le même temps à l'Association des Instituts d'études européennes (AIEE) afin d'étendre son action. Le 19 avril 1958 ses statuts se transformèrent en « statuts universitaires » après délibération du conseil général de l'université de Caen avec l'appui du directeur de l'enseignement supérieur, et par décret du 20 juin 1960. Son appellation devint alors «Centre de recherches et d'études de psychologie des peuples et de sociologie économique », toujours localisé au Havre mais rattaché à l'Université de Caen. L'association liée à l'Institut fut dissoute au début des années 1960 puis refondée en «Société des amis du centre universitaire Havrais » subissant les contre coups économiques et moraux des décolonisations successives ${ }^{78}$.

Document $\mathbf{n}^{\circ} 4$ : Associations pratiquant le prosélytisme colonial au Havre entre 1880 et $1950^{79}$ :

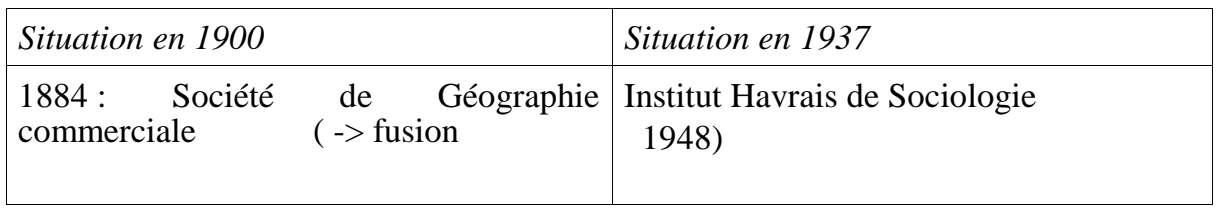




\begin{tabular}{|l|l|}
\hline $\begin{array}{l}1898: \text { Société d'aide et de protection aux } \\
\text { colons (-> fusion }\end{array}$ & $\begin{array}{l}1929: \text { Institut Colonial } \\
1934)\end{array}$ \\
$1904:$ Ligue coloniale (-> 1940) & $\begin{array}{l}1936: \text { Comité régional de propagande } \\
\text { coloniale }\end{array}$ \\
$1905:$ Ligue maritime et coloniale & $1946:$ Comité pour l'Indochine française \\
\hline
\end{tabular}

Document $\mathbf{n}^{\circ} 5$ : Les adhérents de la Société de géographie du Havre (S.G.C.) et de l'Institut Havrais (I.H.S.P.). Liste publiée dans les Bulletins de la Société de Géographie. Adressographe de l'Institut Havrais de 1884 à $1970^{80}$.
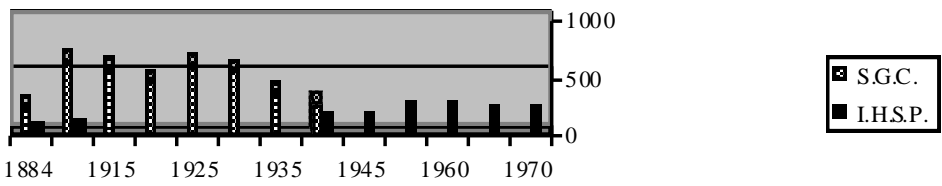

Document $\mathbf{n}^{\circ} \mathbf{6}$ : Les 8 premiers membres de la direction et de l'administration de l'Institut Havrais (1937-1946) ${ }^{81}$.

\begin{tabular}{|l|l|}
\hline Membres & Fonctions \\
\hline $\begin{array}{l}\text { Georges } \\
\text { Vachoux }\end{array}$ & $\begin{array}{l}\text { Secrétaire du Comité de l'industrie, du commerce et de l'armement } \\
\text { du Havre et de l'Estuaire de la Seine ; Dirigeant de la Société de } \\
\text { géographie commerciale et de l'Institut colonial du Havre ; Pré- } \\
\text { sident de l'Institut Havrais de 1937 à 1946. } \\
\text { Louis Hubert }\end{array}$ \\
$\begin{array}{l}\text { Eégociant en café ; Président de l'Institut Havrais en 1946. } \\
\text { Amphoux }\end{array}$ & $\begin{array}{l}\text { Négociant en coton; Membre de l'Institut colonial du Havre ; vice- } \\
\text { président de l'Institut Havrais de 1937 à 1945. }\end{array}$ \\
$\begin{array}{l}\text { Augustin } \\
\text { Normand }\end{array}$ & Armateur en construction navale. \\
\hline $\begin{array}{l}\text { Rodolphe } \\
\text { Rufenacht }\end{array}$ & $\begin{array}{l}\text { Négociant en coton; Dirigeant de l'Institut colonial du Havre et du } \\
\text { Comité régional de propagande coloniale. }\end{array}$ \\
\hline
\end{tabular}




\begin{tabular}{|l|l|}
\hline Albert Charles & $\begin{array}{l}\text { Négociant en bois coloniaux ; Dirigeant de l'Institut colonial du } \\
\text { Havre et du Comité pour l'Indochine française. } \\
\text { Entrepreneur en Bâtiment ; Dirigeant de l'Institut colonial du } \\
\text { Havre. }\end{array}$ \\
Abel Miroglio & \begin{tabular}{l} 
Professeur de philosophie ; Directeur de l'Institut Havrais en 1945. \\
\hline
\end{tabular}
\end{tabular}

\section{4-Les champs de recherche de l'Institut et leurs étapes des années 1940 aux années 1980.}

Les thèmes, qualifiés du temps de la «République coloniale » d'《exotiques », portaient surtout sur «l'ethnologie coloniale» mais aussi sur les «pays neufs » (États-Unis, Brésil) ainsi que sur les états européens partagés depuis 1946 par le « rideau de fer». Ainsi sur 235 conférences données par l'Institut au cours de la période 1945-1962, 42 concernaient le monde colonial (20 l'Afrique noire et Madagascar, 6 l'Afrique du Nord et 2 l'Indochine). Toutes les autres portèrent donc sur l'Europe, l'Amérique du Nord et du Sud ainsi que l'Asie non coloniale. Malgré les études réalisées par l'Institut Havrais des colonies incluses dans l'Union Française il semble n'y avoir eu que peu d'interrogations sur les échecs de l'Empire français. Ceci paraît expliquer en grande partie l'oubli de ces travaux par la suite.

Pourtant les « décades » (cycles de dix conférences tenues essentiellement en été, initiées par l'Institut) rassemblaient de nombreux étudiants Français et étrangers. Une majorité provenait d'ailleurs d'Allemagne et du Bénélux. On note aussi l'absence (relevée par les membres de l'Institut) d'étudiants anglais pourtant relativement proches géographiquement. Dans le même temps, de jeunes professeurs mais aussi de nombreux hommes d'affaires, un public particulièrement visé par l'Institut, se joignaient régulièrement à eux. À noter qu'en 1954, un cycle de conférences accueillit pour principal intervenant Léopold Sédar Senghor, député du Sénégal, qui menait alors une triple carrière d'écrivain, de professeur et d'homme politique. Senghor fut nommé sous-secrétaire d'état en 1955 avant de devenir premier président de la République du Sénégal en 1960. Il prit pour sujet d'exposé à l'Institut Havrais une problématique ayant pour thème «Ce que l'Afrique attend de l'Europe ». Senghor y affirmait que devenue un «immense laboratoire » pour les Européens, l'Afrique n'aurait sans doute rien à demander à l'Europe, si celle-ci n'avait d'abord commencé à l'envahir ${ }^{82}$ : les Européens avaient, selon l'intervenant, fait tarir les cultures vivrières au profit des cultures d'exportation au lieu de mettre un terme à l'économie de traite mais aussi de développer les industries...

Mais, dans les sujets généraux abordés par l'Institut, la construction européenne, l'analyse du monde anglo-saxon, le nouveau visage du monde après les deux guerres mondiales ainsi que l'importance de l'économie maritime européenne semblaient désormais peser davantage sur les préoccupations de ses membres. Il faut aussi remarquer qu'entre l'École Pratique coloniale, l'École Technique de la France d'Outre-Mer et l'Institut Havrais, il existait de nombreux liens qui favorisaient 
toujours la conservation des valeurs coloniales toujours fréquemment répandues parmi les membres de l'Institut.

Document $\mathbf{n}^{\circ} 7$ : «Le Canal de Suez en $1929 »$ article d'André Siegfried paru dans Le Petit Havre du 27 avril 1930.

«Comme l'Empire romain à la fin de sa course, l'empire économique de la race blanche tend donc à se diviser en deux zones distinctes, avec deux têtes dont l'une est en Europe et l'autre aux États-Unis. Les têtes demeurent aussi dans la race blanche et dans l'hémisphère septentrional, mais si jamais les pays exotiques s'industrialisent et revendiquent leur indépendance, alors c'est tout le visage de la planète qui risque de se modifier ».

\section{Document $\mathbf{n}^{\circ} 8$ : Préface d'André Siegfried aux Bois en Côte d'Ivoire ${ }^{83}$} (1954).

«Le sens de l'organisation est le fait du Blanc, on pourrait même dire du Blanc occidental. Bien suggestive est la psychologie que vous dressez, soit de l'Européen, soit du Nègre, dans la mesure où il est utilisable dans les fonctions qu'on est en droit d'attendre de lui. Qu'il s'agisse de souplesse, d'agilité, de précision dans les mouvements, le Noir ne sera pas inférieur. C'est quand il faut faire preuve de sens des responsabilités, de capacité d'organisation, de soin dans la gestion des outillages qu'il se révèle inférieur à l'Européen. Il a besoin de direction, et cette direction c'est le Blanc, le Blanc seul qui peut la donner. Chose curieuse, et que j'ai plusieurs fois observée, c'est qu'alors le dernier des Blancs est encore supérieur comme efficacité au meilleur des Noirs ».

De nombreux spécialistes français et étrangers très divers furent appelés à faire des conférences à l'Institut : Octave Mannoni, Georges Balandier ${ }^{84}$, Albert Nicollet (professeur de géographie et de psychologie d'Outre-Mer), des hommes politiques venus d'horizons différents ${ }^{[85]}$ (Francisque Gay, André Philip, Pierre Mendès France, Pierre-Henri Teitgen, le diplomate André François-Poncet, Paul Van Zeeland, ministre d'État de Belgique, René Coty et deux maires du Havre), mais aussi plusieurs juristes, géographes et sociologues (Charles Baudoin, René Le Senne, Gaston Berger, Gabriel Le Bras, Roger Bastide, Raymond Aron, l'auteur en 1962 de Paix et Guerre entre les nations, et toujours A. Siegfried). Cette effervescence intellectuelle recevait un écho certain dans la presse locale. Six « décades » (cycles de conférences), furent organisées de 1949 à 1965. 1300 lettres étaient adressées chaque année à l'Institut.

Entre 1946 et 1970, le réseau de ses correspondants s'étendait à environ 120 personnes d'après une liste publiée en $1958^{86}$. Il restait composé en majorité de professeurs de l'enseignement supérieur. En 1966 le C.E.R.I.N (« Centre d'études des relations interethniques de Nice»), centre déjà spécialisé sur les enjeux 
d'immigration, apporta une documentation importante à l'Institut Havrais ${ }^{87}$. Vers la fin des années 1970, Miroglio put planifier son vaste Dictionnaire des populations européennes publié définitivement en 1978. Ce dernier rassemblait 258 articles ayant pour sujet principal les populations urbaines et les identités collectives. Ses contributions avaient été écrites par 168 auteurs provenant de 18 états européens ${ }^{88}$. Le fameux dictionnaire devint l'ouvrage principal de «l'école de psychologie des peuples » organisée au Havre alors que l'Institut devait trouver matière à se «ressourcer» suite aux décolonisations massives et successives à l'intérieur de l'ancien Empire colonial français. Traitant d'une Europe qui s'étendrait de la Bretagne à l'Oural et de la Galice à la Mer Noire, les auteurs du dictionnaire se prononçaient pour l'avènement d'une Europe plutôt Confédérale, une aspiration portée par plusieurs intellectuels libéraux depuis l'après seconde guerre mondiale tels André Siegfried ou Pierre-Henri Teitgen ${ }^{89}$. Mais bien avant déjà, Miroglio avait organisé deux colloques, l'un en 1948, et l'autre, en 1955 à Nice dans le cadre du Centre Méditerranéen et, désormais, son but prioritaire était d'assoir la «psychologie des peuples » à un niveau scientifique et interdisciplinaire: nature, fondements, objectifs et subjectifs, des identités collectives, un vaste et long programme pour tous les collaborateurs de l'Institut. En 1952 un concours de «l'Institut » fut même établi avec un prix de 100000 francs. Le jury était composé alors de 7 personnes dont quatre Français (en permanence parmi eux Siegfried et Miroglio), ainsi que trois personnalités étrangères. Jusqu'à son décès en 1959, l'Institut reçut l'appui régulier d'André Siegfried, devenu le «Pape» de la Science-Politique française. Ainsi, ce dernier n'hésita pas à organiser des manifestations parisiennes, notamment à Science-Po, en invitant le directeur du Port autonome du Havre ainsi que le spécialiste des relations extérieures de sa Chambre de commerce. En outre, à partir de 1960, l'Institut devint un centre de recherche qui pouvait donner des diplômes de $3^{\mathrm{e}}$ cycle (sous la direction de Miroglio). D'ailleurs sa femme avait traduit le petit ouvrage d'anthropologie-culturelle de Margaret Mead Themes in French Culture (Thèmes culturels en France) qu'elle avait publié précédemment avec un ensemble critique de sept personnalités dont André Siegfried, Gabriel Le Bras et Jean Stoetzel, fondateur de la Revue Française de Sociologie en $1960^{90}$. Dans le contexte de la guerre froide, des nouvelles luttes d'influence et relations internationales, le principal réseau des correspondants internationaux de l'Institut se trouvait curieusement situé au Bénélux et en Allemagne. Dans le même temps, la forte association havraise «France- Grande-Bretagne» semblait délaissée malgré les rencontres de Southampton qui marquèrent l'Institut (quelques années avant les journées franco-néerlandaises organisées au Havre en 1950). Ainsi, bien que des liens aient été contractés depuis longtemps avec cette « vieille puissance coloniale », les oppositions intra-européennes pouvaient peser sur son recrutement depuis que la France avait mis son veto à l'entrée du Royaume-Uni dans la C.E.E (Communauté Economique Européenne) en janvier 1963.

Document $\mathbf{n}^{\circ} \mathbf{9}$ : Les sept premiers présidents d'honneur de l'Institut.

Liste

André Siegfried

Fonction honorée par l'Institut

Membre de l'Académie Française 


$\begin{array}{ll}\text { Marcel Hérubel } & \text { Membre de l'Académie de Marine } \\ \text { Charles Rist } & \text { Economiste libéral } \\ \text { Paul Van Zeeland } & \text { Homme politique belge } \\ \text { Gabriel Le Bras } & \text { Juriste } \\ \text { René Coty } & \text { Homme politique français }\end{array}$

Entre 1970 et 1982, ce fut l'Ethnopsychologie qui prit donc le dessus sur les autres champs disciplinaires, avec à la tête de l'Institut, Bernard Guillaumin, maître de conférences en philosophie. Le comité de rédaction de la revue rassemblait alors des spécialistes en psychologie sociale, en littérature et civilisations, en psychanalyse, en sociologie et en anthropologie africaine. Le caractère interdisciplinaire impressionnant s'imposa au travers des colloques de 1970, 1971 et 1973. Mais, la crise de l'Institut se fit jour en 1971 : l'Université de Rouen refusa d'allouer de nouveaux crédits au centre de recherche qui dut disparaître temporairement à partir de 1978. Il fut finalement recréé grâce au soutien de la Chambre de commerce du Havre puis rattaché au groupe de recherche «Philosophie et Anthropologie » de l'Université de Rouen. Les Cahiers de Sociologie économique parus de 1959 à 1966, puis de 1971 à 1972, reparurent de 1979 à 1982. Ils furent transformés sous la direction d'Albert Nicollet en Cahiers de Sociologie économique et culturelle en 1984, dans une dynamique de recherche initiée par l'Association pour la Recherche en «Ethno-Psycho-Sociologie» à partir de 1974. L'Institut continua cependant avec difficulté à trouver sa place au sein des sciences sociales tout comme sa nouvelle revue remaniée de «psychologie des Peuples » ${ }^{91}$. Son écho parut alors limité.

\section{Conclusion}

Après sa fondation en 1937 à la fin de la III $^{\mathrm{e}}$ République l'Institut Havrais eut une première période d'apothéose de 1945 à 1965 puis une seconde de forte décrue liée à l'échec de l'Union Française mais aussi, en toile de fond, aux deux guerres coloniales menées par la France (Indochine, Algérie). La période ouverte après la seconde guerre mondiale fut aussi celle de l'affirmation des «nations » et des «peuples» du «Tiers-Monde» (selon l'expression forgée justement par Alfred Sauvy). D'un côté, les problèmes liés au développement des continents colonisés ainsi qu'à la disparition des empires coloniaux et, de l'autre, la recherche d'unité à l'échelle européenne pour préserver la paix après 1945, formèrent le contexte essentiel du rayonnement de l'Institut Havrais. Enfin, la société française avait été bouleversée dans ses fondements mêmes par la tertiarisation (et l'urbanisation) avancée issue des «Trente glorieuses ». La revue de «Psychologie des peuples», qui touchait jusqu'à une soixantaine de pays dont le Japon, l'Australie et la Nouvelle-Zélande, dut se revivifier avec la mise en place de nouveaux «Cahiers de Sociologie économique » à partir de 1959. Son succès était alors surtout local à la différence des années antérieures. Ces cahiers furent à nouveau rebaptisés à la fin des années 1970 «Ethnopsychologie» soutenus à ce moment par le sociologue 
Gurvitch, fondateur de l'Association internationale des sociologues de langue française (en 1956). Gurvitch était le principal opposant à la fois de la sociologie américaine et des grands systèmes théoriques issus du durkheimisme, du marxisme et du structuralisme ${ }^{92}$. La revue comptait, à cette époque, environ 600 abonnés notamment aux Etats-Unis. Mais, en retour de ses abonnés, l'Institut recevait environ 80 revues étrangères en sciences humaines. Il conservait aussi un correspondant dans chaque université française. Dans ces nouvelles conditions, trois axes majeurs voulurent être mieux explorés par les collaborateurs de l'Institut en relation avec des thématiques plus novatrices: le Tiers-Monde et les villes nouvelles dont le Havre mais aussi la «géo-sociologie » du monde maritime.

Les sources de l'Institut havrais « entre science et politique » restent à ce jour trop peu connues, pourtant leur analyse ciblée apporterait de nombreuses indications sur le rôle et la place des ses acteurs, autour des notions d' "ethnopsychologie » voire d' « anthropologie » liée à la « géopolitique » (« la géographie, ça sert d'abord à faire la guerre » remarquait, entre autres, Y. Lacoste en 1976, mais du commerce aussi..., de la psychologie et vice versa...). Elle permettrait aussi de s'interroger sur la notion très subjective, donc particulièrement discutable, de «psychologie des Peuples »: paradigme né au tournant du $\mathrm{XIX}^{\mathrm{e}}-\mathrm{XX}^{\mathrm{e}}$ siècles puis qui se diffuse en France de la veille de la seconde guerre mondiale à l'après décolonisation.

Les archives de l'Institut et les sources locales mériteraient des recherches plus pointues sur son fonctionnement, ses idées, leur diffusion et leur réception en France et à l'étranger tout en les liant à l'essor des nouvelles «sciences humaines ». Elles permettraient un examen de ses réseaux, de leur ampleur et de leur consistance, rattachés à la fois au contexte économique et géographique du port havrais mais aussi aux nouveaux enjeux des relations internationales: le déclin de l'Empire colonial français, puis, la longue période de la guerre froide et du conflit entre les blocs (1945-1989). Dans cette perspective on peut se demander si les influences directes (et indirectes) des travaux mais aussi de la personnalité du «grand havrais » A. Siegfried pour l'essor de cette « institution » et sa «science nouvelle», la «psychologie des Peuples », ne furent pas fondamentales?

\section{SOURCES}

\section{Archives départementales de la Seine-Maritime}

Série 4 M 574 : propagande pour la colonisation.

Série 4 M 575 : Statuts de l'Institut Havrais de Sociologie économique et de psychologie des peuples (Déclaration du 15 février 1938).

Cote : JPL 239 : Revue de psychologie des peuples (1953-1970) puis Ethnopsychologie. Revue de psychologie des peuples (1970-1989) et Cahiers de Sociologie économique et culturelle (après 1989).

Cote : JPL 239/7 : Nicollet Albert (1999), Un homme, une ville, une ouvre: Abel Miroglio (1895-1978), Le Havre, Institut Havrais de Sociologie, 127p. (Repris des Cahiers de Sociologie économique et culturelle, $\mathrm{n}^{\circ}$ hors-série 1999).

\section{Bibliothèque municipale du Havre}


Revues :

- Bulletin de l'Institut colonial du Havre.

- Bulletin de la Société de Géographie commerciale du Havre (1899->).

- Le Havre-Colonial (avril-juin 1954).

- Le Petit Havre (année 1947).

- Revue de la Porte Océane. (dont le directeur est membre de l'Institut Havrais).

Dossier 92 N : André Siegfried, 1875-1959, géographe.

- Miroglio A. (sans date, 1985 ?), «Un grand havrais. André Siegfried », Bulletin Maritime du Havre, $20 \mathrm{p}$.

- _ (1975), «L'œuvre d'A. Siegfried. Le pionnier de la sociologie politique » et

«Le grand témoin de notre époque » dans La vie Havraise, Jeudi 29 mai et $1^{\text {er }}$ juin.

- prospectus du Comité pour le Centenaire de la naissance d'André Siegfried (Paris 10 janvier 1985).

- bibliographie des œuvres d'A. Siegfried.

- «A. Siegfried et la démocratie britannique»(1979), Le Havre-Presse, 26 novembre.

- « Jules Siegfried aux Etats-Unis » (1955), Livres de France, n², février.

Dossier 92 N : Miroglio Abel (1895-1978), philosophe.

Cote : PN. 946: Revue de psychologie des peuples puis Ethnopsychologie. Centre Havrais de psychologie des peuples et de sociologie économique 1946->1974.

\section{Bibliothèque municipale de Rouen}

DUROSELLE J.-B. (1975), Discours de la célébration du centenaire de la naissance d'André Siegfried, Paris, Firmin-Didot, 35 p. Cote : Brmm 2022-1975-10.

\section{Fonds des Archives d'Histoire contemporaine de Science-Po}

Fonds André Siegfried : il rassemble 94 cartons (ils ont largement été dépouillés et exploités par l'historien Pierre Birnbaum ${ }^{93}$ ). Le fonds est référencé au sein de plusieurs inventaires.

Institut havrais de Sociologie économique et culturelle, 56 rue Anatole France, Le Havre, 76600 (centre qui existe encore aujourd'hui).

On consultera aussi utilement les ouvrages et articles de référence suivants à caractère de source sur la tradition historiographique française des études de «psychologie politique » des débuts de la III ${ }^{\mathrm{e}}$ à la IVe République :

\section{Les régions}

BERR H. (1903), «La synthèse des études relatives aux régions de la France » dans Revue des Sciences Historiques, $\mathrm{n}^{\circ}$ 6, p. 177-178.

LORQUET P. (1901), «Quels cadres choisir pour l'étude psychologique de la France ? » dans Revue des Sciences Historiques, $\mathrm{n}^{\circ} 2$.

PONCIN P. (1900), «Introduction à l'étude des régions de France » dans Revue des Sciences Historiques, $\mathrm{n}^{\circ}$ 1, p. 14-20.

SIEGFRIED A. (1913), Tableau politique de la France de l'Ouest sous la III République, Paris, A. Colin, 1964, 535 p. 
VIDAL DE LA BLACHE P. (1914), « Compte-rendu du Tableau Politique de la France de l'Ouest » dans Annales de Géographie, vol. 23, n 129, p. 261-264.

\section{Les peuples \\ Études en français}

BARDOUX J. (1906-1907), Essai d'une psychologie de l'Angleterre contemporaine, Paris, Alcan, vol. 1 «Les crises belliqueuses », 563 p. et vol. 2, «Les crises politiques : protectionnisme et radicalisme », 291 p. (publié dans la nouvelle collection «Peuples et Civilisations » dirigée par Sagnese et Halphen)

BOUTMY E. (1901), Essai de psychologie politique du peuple anglais au XIX ${ }^{e}$ siècle, Paris, A. Colin, $456 \mathrm{p}$.

- (1902), Éléments d'une psychologie politique du peuple américain. La Nation, la patrie, l'Etat, la religion, Paris, A. Colin, 366 p.

FOUILLEE A. (1898), Psychologie du peuple français, Paris, Alcan, 391 p.

- (1902), Esquisse psychologique des peuples européens, Paris, Alcan, 552 p.

HARDY G. (1939), La géographie psychologique, Paris, Gallimard, Coll. Géographie Humaine, $188 \mathrm{p}$.

- (1947), «La psychologie des populations coloniales, état présent de la question » dans Revue de Psychologie des Peuples, p. 233-261.

LE BON G. (1978), Lois psychologiques de l'évolution des peuples, Paris, Les Amis de G. Le Bon, Rééd., 176 p.

LETOURNEAU Ch. (1884), La sociologie d'après l'ethnographie, Paris, Rinwald, $608 \mathrm{p}$.

- (1895), La guerre dans les diverses races humaines, Paris, L. Bataille, 587 p.

- (1901), La psychologie ethnique, Paris, Schleischer Frères, 556 p.

MIROGLIO A. (1978) (S. Dir.), L'Europe et ses populations : vues d'ensemble et dictionnaire descriptif, La Haye, M. Nijhoff, 828 p.

SIEGFRIED A. (1932), « La psychologie allemande et l'attitude allemande à l'égard de quelques grands problèmes ", conférences faite à la Société d'enseignement scientifique, le 27/01/1933 dans Bulletin de la Société de Géographie commerciale, p. 33-56.

- (1943), Vue générale de la Méditerranée, Paris, Gallimard, 191 p.

- (1948), L'Afrique du Sud. Notes de voyages, Paris, A. Colin, 160p. Série d'articles publiés dans le Figaro (4 mois d'enquête au Congo Belge, en Rhodésie et en Afrique du Sud).

- (1950), L'âme des peuples, Paris, Hachette, 221p. (exemplaire dédicacé par l'auteur à M. Abel Miroglio, conservé à la Bibliothèque municipale du Havre Cote : R. I 476).

- (1955), «Psychologie du Normand » dans Études Normandes, p. 233-241.

TEILlaC J. (1954) (Préface d'A. Siegfried), Les bois de la côte d'Ivoire, leurs conditions d'exploitation et de vente, Paris, Ed. maritimes et coloniales, 88 p.

\section{Études en anglais}

SIEGFRIED A. (1906), Le Canada : les deux races, Problèmes politiques contemporains, Paris, Colin, 415p. [Traduction anglaise: The race question in Canada (1966), Ottawa, The Carleton Librairy]

—_ (1927), Les États-Unis d'aujourd'hui, Bibliothèque du Musée Social, Paris, Colin, 362 p. 
[Traduction anglaise : America Comes of Age. A French Analysis, New York].

- (1930), France. A study in Nationality, New Haven, Yale University Press, 122 p. [Série de conférences universitaires].

- (1951), "Approaches to an understanding of modern France" dans Earle E.M., e.d., Modern France, Princeton University Press.

\section{BIBLIOGRAPHIE}

\section{L'essor de la psychologie des peuples}

Duroselle J.-B. (1990), L'Europe. Histoire des peuples, Paris, Perrin, Pluriel, $705 \mathrm{p}$.

GotTMANN J. (1952), La politique des états et leur géographie, Paris, A. Colin, Coll. Sciences Politiques, 228 p.

SAUVY A. et LEDERMANN S. (Juillet-Septembre 1946), "La guerre biologique (1933-1945) : population de l'Allemagne et des pays voisins » dans Population, $\mathrm{n}^{\circ}$ 3 , p. 471-488.

\section{Contexte culturel, national et politique}

AZEMA J.-P. et WIEVIORKA O. (2004), Vichy 1940-1944, Paris, Perrin, 374 p.

BIRNBAUM P. «Accepter la pluralité : haine et préjugés » dans Histoire des droites en France (Dir. J.-F. Sirinelli), Paris, Gallimard, 1992, t.3 «Sensibilités », p. 423471.

FAVRE P. (1989), Naissance de la science politique en France 1870-1914, Paris, Fayard, 331 p. (notamment la $4^{\mathrm{e}}$ partie, p. 233-306).

- (1981), «Les sciences d'État entre déterminisme et libéralisme. Emile Boutmy (1835-1906) et la création de l'Ecole Libre des Sciences Politiques » dans Revue Française de Sociologie, vol. 22, p. 429-465. (fondamental sur le rôle de la famille Siegfried et des réseaux protestants dans la fondation nationale des sciences politiques).

LEQUIN Y. (2006), Histoire des étrangers et de l'immigration en France, Paris, Larousse, $544 \mathrm{p}$.

MAYEUR J.-M. (1973), Les débuts de la troisième République 1871-1898, Point Seuil, Histoire, 256 p.

- (1984), La vie politique sous la III République (1870-1940), Paris, Point Seuil, Histoire, 449 p.

MILZA P. (1987), Fascisme français : passé et présent, Paris, Flammarion, 463 p.

NICOLET Cl. (2006), La fabrique d'une nation. La France entre Rome et les Germains, Paris, Perrin, 361 p.

NOIRIEL G. (2007), Immigration, antisémitisme et racisme en France (XIXe-XXe siècle). Discours publics, humiliations privées, Paris, Fayard, 715p.

- (1999), Les origines républicaines de Vichy, Paris, Hachette, 335 p.

RIOUX J.-P. (1980), La France de la Quatrième République, t. 1 « L'ardeur et la nécessité 1944-1952 », Point Seuil, Histoire, 314 p.

ROUSSO H. (1990), Le syndrome de Vichy de 1944 à nos jours, Paris, Point Seuil, Histoire, $417 \mathrm{p}$.

WINOCK M. (2004), Nationalisme, antisémitisme et fascisme en France, Paris, Point Seuil, Histoire, 418 p.

ZELDIN Th. (2002), Histoire des passions françaises (1848-1945), Paris, Petite Bibliothèque Payot, 5 vol. 


\section{Contexte économique et colonial}

AGERON Ch. R. (1991), La décolonisation française, Paris, A. Colin, 179 p.

ARENDT Hannah (1968), Les origines du totalitarisme. L'impérialisme, Fayard, 350 p. (notamment les chapitres « Race et bureaucratie » p. 111-170 ; «Le déclin de l'État-Nation et la fin des Droits de l'homme », p. 239-292.)

BANCEL N., BLANCHARD P., VERGES F. (2003), La République coloniale, Paris, Pluriel, $174 \mathrm{p}$.

BLANCHARD P. et LEMAIRE S. (2004), Culture impériale 1931-1961. Les colonies au cœur de la république, Paris, Autrement, 276 p.

GIRARDET R. (1972), L'idée coloniale en France de 1871 à 1962, Paris, Hachette, $506 \mathrm{p}$.

GIRAULT R. et FRANK R. (1997 et 1998), Diplomatie européenne. Nations et impérialismes 1871-1914 et Turbulentes Europe et nouveaux mondes 1914-1941, Paris, Petite Bibliothèque Payot, T. I et II, 451 p. et 514 p.

- et THOBIE J. (2005), La loi des géants, 1941-1964, Paris, Petite Bibliothèque Payot, T. 3, 541 p.

LEJEUNE D. (1993), Les sociétés de géographie en France et l'expansion coloniale au XIX $X^{e}$ siècle, Paris, A. Michel, 236 p.

MANCERON G. (2003), Marianne et les colonies, une introduction à l'histoire coloniale de la France, Paris, La Découverte, 317 p.

MARSEILlE J. (1986), L'âge d'or de la France Coloniale, Paris, A. Michel, 144 p.

- (1986), Empire colonial et capitalisme français : histoire d'un divorce, Paris, A. Michel, $461 \mathrm{p}$.

VAÏSSE M. (1991), Les relations internationales depuis 1945, Paris, A. Colin, $190 \mathrm{p}$.

\section{Contexte colonial havrais}

La mer et l'Empire, textes d'André Siegfried et alii, (1943) conférences faites à l'Institut maritime et colonial, $228 \mathrm{p}$.

LEGOY J. (1982 et 1984), Le peuple du Havre et son histoire, Du négoce à l'industrie : 1800-1914, t. 2, Le cadre de vie, 447 p. et t. 3, La vie politique et sociale, $511 \mathrm{p}$.

MALON Cl. (1997), «Le Havre et l'outre-mer. Sociabilité et recherche. Les origines et le développement de l'Institut havrais. Le contexte colonial » dans Etudes Normandes, $\mathrm{n}^{\circ} 2$, p. 75-96.

- (2006), Le Havre colonial de 1880 à 1960, Presses Universitaires de Rouen et du Havre, notamment p. 572-575.

- (1996), Réseaux et stratégies du Havre colonial, D.E.A. (S. Dir. D. Barjot), Université de Caen.

NICOLLET (A.) (1996), «La longue marche de la sociologie économique à l'Institut havrais de sociologie » dans Cahiers de Sociologie économique et culturelle- Ethnopsychologie, vol. 26, décembre, p. 91-121.

SIEGFRIED (A.) (1904), Edward Gibbon Wakefield et sa doctrine de la colonisation systématique, thèse pour le doctorat, Paris, Colin, 1904, 116p. (le premier ouvrage publié par A. Siegfried, fondamental pour la compréhension de ses centres d'intérêts).

\section{Le Havre politique et culturel}


ARDAILlOU P. (1999), Les républicains du Havre au XIX siècle (1815-1889), Presses universitaires du Havre et de Rouen, 452 p.

LEGOY J. (1986), Cultures havraises 1895-1961, Ed. EDIP, Saint-Étienne du Rouvray, $367 \mathrm{p}$.

MIROGLIO A. (1949), La vie intellectuelle au Havre, L'opinion, $\mathrm{n}^{\circ}$ du 10 novembre. - (1967), «Témoignages sur le Havre » dans Revue de Psychologie des Peuples, $\mathrm{n}^{\circ} 1, \mathrm{t} .22$.

NICOLLET A. (2003), « La construction d'une identité de la Normandie dans la première moitié du XX ${ }^{\mathrm{e}}$ siècle » dans Etudes Normandes, 2003, vol.52, n 1, p. 11-24.

RENEAU S. (1990), «Politique et pratiques culturelles au Havre, 1944-1965 » dans Études Normandes, $\mathrm{n}^{\circ} 1$, p. 7-22.

\section{La psychologie des peuples : une synthèse républicaine ?}

La psychologie des peuples et ses dérives, Journées d'études organisée par le Groupe d'études pluridisciplinaires d'histoire de la psychologie (1999), Paris, 3-5 juin 1996, Ed. M. Kail et G. Vermès, C.N.D.P., 205 p.

CLARET Ph. (1999), «Psychologie collective, psychologie des peuples et Science politique en France depuis 1870 : histoire d'un rendez-vous manqué » dans La psychologie des peuples et ses dérives, Op. Cit., p. 63-80.

LE QUINTREC G. (1991), « Alfred Fouillée et la psychologie des peuples », Cahiers de Sociologie, Economique et Culturelle, $\mathrm{n}^{\circ}$ 15, juin, p. 35-60. (article issu d'un mémoire de D.E.A., Dir. P. Nora, E.H.E.S.S, 1989).

OZOUF J. et OZOUF M. (1984), «Le tour de France par deux enfants, Le petit livre rouge de la République » dans Les lieux de mémoire (S. Dir. P. Nora), t. 1, La République, Paris, Gallimard, p. 291-321.

ROSANVALLON P. (2000), La démocratie inachevée. Histoire de la souveraineté du peuple en France, Paris, Gallimard, 591 p.

- (1998), Le peuple introuvable. Histoire de la représentation démocratique en France, Paris, Gallimard. (essentiellement le chapitre "Sociologie et démocratie », p. 130-175.)

STERNHELL Zeev (2000), « Morphologie et historiographie du fascisme en France » dans Ni droite, Ni gauche. L'idéologie fasciste en France, Paris, Fayard, p. 11-112. (Préface à la $3^{\mathrm{e}}$ édition).

- (1997), La droite révolutionnaire 1885-1914. Les origines françaises du fascisme, Paris, Gallimard, $602 \mathrm{p}$.

THIEC Y. (1981), «Gustave Le Bon, prophète de l'irrationalisme » dans Revue Française de Sociologie, vol. 22, n 3, p. 409-428.

\section{Le concept de personnalité nationale (National Character)}

CLARET Ph. (1993), La notion de personnalité nationale. Essai d'analyse comparée des théories modernes françaises et anglo-saxonnes, thèse de doctorat d'État en Science politique, Université de Bordeaux I, 539 p. (Prix Montesquieu 1993 d'Histoire des idées politiques).

- (1998), La personnalité collective des nations. Théories anglo-saxonnes et conceptions françaises du caractère national. coll. Organisations internationales et relations internationales, $n^{\circ} 40,464 p$.

- (2001), «Theories of National Personnality Revisited : Anglo-American Models and French conceptions » dans Modern Roots Studies of National Identity. 


\section{Sociologie, Psychologie des foules et psychologie des peuples}

BARROWS S. (1990), Miroirs déformants : réflexions sur la foule en France à la fin du $X X^{e}$ siècle, Paris, Aubier, $226 \mathrm{p}$.

BEJIN A. (1987), «Théories socio-politique de la lutte pour la vie » dans Nouvelle Histoire des idées politiques (S. Dir. P. Ory), Paris, Hachette, p. 321-331.

GAUCHET M. (1992), «La pensée, le langage et la foule » dans L'inconscient cérébral, Paris, Seuil, p. 105-126.

MOSCOVICI S. (1985), L'âge des foules. Un traité historique de psychologie des masses, Paris, Complexe, 503 p.

NYE ROBERT A. (1975), The Origins of crowd psychology. Gustave Le Bon and the Crisis of Mass Democracy in the third republic, London/Beverly Hills : Sage, 248 p.

STERNHELL Zeev (1978), La droite révolutionnaire. Les origines françaises du fascisme 1885-1914, Paris, Le Seuil, 441 p.

THIEC Y. (1982), Gustave Le Bon, la psychologie des foules, la fondation de la psychologie collective et sa propagation dans les sciences sociales à la fin du XIX ${ }^{e}$ siècle, Institut universitaire européen, thèse de doctorat, 1982.

\section{Psychologie des peuples, Nation et « géographie des races »}

BALIBAR E. et WALLERSTEIN E. (1997), Race, nation, classe : les identités ambiguës, Paris, La Découverte, 1997, 307 p.

Des sciences contre l'homme (S. Dir. Cl. Blanckaert), vol.II, « Au nom du bien », Ed. Autrement, Paris, 1993, 147p.

GUIRAL P. et TEMINE E. (1977) (S. Dir.) (Recueil d'articles présenté par), L'idée de race dans la pensée politique française contemporaine, Paris, Ed. du C.N.R.S., 281 p. notamment le chapitre II «Idée de race et pensée politique française (Pensée de gauche et de droite) au XIX ${ }^{\mathrm{e}}$ siècle », p. 34-156.

LEBRAS G . (1966), «Psychologie des peuples et histoire » dans Revue de psychologie des peuples, 121, $\mathrm{n}^{\circ} 1$, p. 21-28.

LELANNOU M. (1961), «La géographie est-elle une science politique » dans Revue française de Science politique, vol. 11, 1961, p. 809-818.

LEVI-STRAUSS Cl. (1987), Race et Histoire, Paris, Denoël, 1987, 127 p.

LIAUZU Cl. (1992), «Psychologie des peuples et des races » dans Race et civilisation. L'autre dans la culture occidentale. Anthologie critique, Paris, Syros, 492p. (notamment Quotient intellectuel, Psychologie et races, p. 108-120).

MICHAUD G. (1978), Identités collectives et relations inter-culturelles, Ed. Complexes, $249 \mathrm{p}$.

MUCCHIELLI L. (1997), « Psychologie des peuples. Région, race et milieu social. Problèmes scientifiques et enjeux disciplinaires d'une théorie de l'histoire autour d'Henri Berr et de la Revue de synthèse historique (1890-1925)» dans Biard A., Bourel D. et Brian E., Henri Berr et la culture du XX $X^{e}$ siècle, Paris, Albin Michel, p. 81-110.

NOIRIEL G. (2001), Etat, nation et immigration. Vers une histoire du pouvoir, Paris, Gallimard, $587 \mathrm{p}$.

- (1988), Le creuset français. Histoire de l'immigration XIX $X^{e}-X X^{e}$ siècles, Paris, Seuil, $441 \mathrm{p}$.

STERNHELL Z. (1977), «Le déterminisme physiologique et racial à la base du nationalisme de Maurice Barrès et de Jules Soury » dans Guiral P. et Témine E. (S. Dir.), op. cit., Paris, Ed. du C.N.R.S., p. 117-138. 


\section{Sur André Siegfried, sa famille et ses origines havraises Jules Siegfried}

ARDAILLOU P. (1992), «Jules Siegfried, un protestant au service d'une république modérée et sociale » dans Cahiers Havrais de Recherche Historique, p. 74-92.

Chambelland C. (1998), (S. dir.), Le Musée Social en son temps, Paris, Presses de l'École Normale Supérieure, 402 p.

MERLIN R. (1923), Jules Siegfried. Sa vie, son oeuvre, Paris, Musée Social, (Bibliothèque municipale du Havre, Cote : N. 2737).

SIEGFRIED A. (1946), Mes souvenirs de la III République : mon père en son temps. Jules Siegfried 1836-1922, Paris, Ed. Du Grand Siècle, 150 p.

VALLEE Th. (1910), Quarante ans de vie républicaine. Jules Siegfried, Le Havre, Journal du Havre. (Bibliothèque municipale du Havre, Cote : N. 2554).

\section{Jacques Siegfried}

MAFFRE Ph. (1988), «Jacques Siegfried, patron de l'enseignement commercial supérieur » dans $R H M C, \mathrm{n}^{\circ} 4$, p. 594-613.

\section{André Siegfried}

ANDLER Ch. (1932), Vie de Lucien Herr (1864-1926), Paris, Rieder, 336 p.

CABANEL P. (1997), «André Siegfried et la Méditerranée : le travail empaysagé et le chasseur de frontières» dans Cahiers de la Villa Kérilos, n ${ }^{\circ}$, p. 175-192.

BIRNBAUM P. (1993), « André Siegfried. La géographie des races » dans La France aux Français. Histoire des haines nationalistes, Paris, Seuil, p. 145-186.

BONNEFOUS E. (1975), «Le centenaire d'André Siegfried» dans Nouvelle Revue des Deux-Mondes, $\mathrm{n}^{\circ}$ 7, p. 17-23.

BONNEFOUS E. et BOIS P. (1977), L'œuvre scientifique d'André Siegfried, Les Presses de Science-Po, Paris, 134 p.

BOULET F., André Siegfried : un maître à penser du XX siècle, mémoire déposé au centre d'histoire de Science-Po, $25 \mathrm{p}$.

BULEON P. (1989), «Siegfried, la Normandie et les tempéraments politiques. Quelques raisons d'une redécouverte » dans Actes du colloque de 1988, Études Normandes, op. cit.

CHEVALIER L. (1977), «André Siegfried et Paris » dans L'œuvre scientifique d'André Siegfried, op. cit., p. 99-111.

CLARET Ph. (1995), «André Siegfried et la psychologie politique. Contribution à la relecture d'une œuvre scientifique » dans Cahiers de sociologie économique et culturelle. Ethnopsychologie, $\mathrm{n}^{\circ} 23$, juin, p. 9-33.

CLAVAL P. (1989), «André Siegfried et les démocraties anglo-saxonnes » dans Études Normandes, $\mathrm{n}^{\circ} 2$.

Célébration du centenaire de la naissance d'A. Siegfried (1975), séance du 26 mai, Institut de France, Académie Française, Section des Sciences Morales et politiques, Paris, $37 \mathrm{p}$.

Comité pour le centenaire de la naissance d'André Siegfried, (1977) Collège de France, Paris, mars 1975, L'œuvre scientifique d'André Siegfried, Presses de la Fondation nationale des Sciences politiques, $129 \mathrm{p}$.

FREMONT A. (1987), «Relire Siegfried en Normandie aujourd'hui » dans Etudes Normandes, $\mathrm{n}^{\circ} 4, \mathrm{n}^{\circ}$ spécial Géopolitique de la Normandie. 
GARRIGOU A. (1995), «L'initiation d'un initiateur. André siegfried et le Tableau politique de la France de l'Ouest», Actes de la Recherche en Sciences Sociales, $\mathrm{n}^{\circ}$ 106-107, mars, p. 27-41.

GOGUEL F. (1959), «En mémoire d'André Siegfried» dans Revue française de Science politique, vol. IX, juin, $\mathrm{n}^{\circ} 2$, p. 333-339.

Hommage à André Siegfried, Textes des allocutions (1961), Paris, Association André Siegfried, F.N.S.P., 59 p.

«L'ouest politique 75 ans après Siegfried » (1987), Géographie Sociale, n 6, Centre de publication de l'Université de Caen, octobre, $312 \mathrm{p}$.

MiLZA P. (1995), Présentation, Siegfried André, Tableau politique de la France de l'ouest, Imp. Nat., 1913, Rééd., Paris, p. 7-35.

MIROGLIO A. (1945), «L'œuvre sociologique de M. André Siegfried » dans Revue de Métaphysique et de Morale, vol. 50, $\mathrm{n}^{\circ} 4$.

MIROGLIO A. (1978), «Un grand havrais, André Siegfried » dans Études Normandes, $\mathrm{n}^{\circ} 2-3$, vol. 27, p. 47-61.

NiCOLLET A. (1989), «André Siegfried et Le Havre» dans Études Normandes, $\mathrm{n}^{\circ} 2$, p. 37-49.

POMMIER J. (1961), Notice sur la vie et les travaux d'André Siegfried (1875-1959), Paris, Institut de France, Firmin-Didot, 24 p.

SANGUIN L. (1985), «André Siegfried et la géographie politique » dans Études Normandes, $\mathrm{n}^{\circ}$ 2, vol. 34, p. 63-68.

WYLIE L. (1977), «André Siegfried à Harvard» dans L'œuvre scientifique d'A. Siegfried, F.N.S.P., p. 67-88.

\section{La géographie politique}

Actes du colloque André Siegfried, la politique et la géographie (1989) Paris-Sorbonne, décembre 1988, Études Normandes, n 2-1989, 136 p. (Sous la présidence de F. Goguel de la F.N.S.P. et A. Lancelot, directeur de l'I.E.P. de Paris, organisé par les géographes P. Claval et Y. Lacoste). (notamment « André Siegfried, l’homme et le milieu », p. 7-48).

BUTTIMER Ann (1971), Society and Milieu in the French Geographic Tradition, Washington, Association of American Geographers, Monograph Series n ${ }^{\circ}$ 6, 226 p.

CLAVAL P. (1977), «Les aspects modernes de la géographie politique » dans Travaux de l'Institut de géographie de Reims, $\mathrm{n}^{\circ}$ 29-30, p. 11-29.

CLAVAL P. (1983), «Le renouveau de la géographie politique » dans Bulletin de l'Association des Géographes Français, 1983, vol. 60, n 493, p. 87-98.

LACOSTE Y. (1984), «Les géographes, l'action et la politique » dans Hérodote, $\mathrm{n}^{\circ}$ 33-34, p. 3-32.

- (1986) (S. Dir.), Géopolitique des régions françaises, Fayard, 3 vol., 1372 p. [approche géopolitique des régions françaises, voir le vol. 2 pour la « Haute-Normandie »].

LEBRAS H. (2006) (entretiens), Entre deux pôles. La démographie entre science et politique, Paris, Aux lieux d'être, 127 p.

MOREAU DEFARGES Ph. (2005), Introduction à la géopolitique, Paris, Seuil, 2005, $252 \mathrm{p}$.

SANGUIN A.-L. (1976), La géographie politique, bibliographie internationale, Montréal, Presses de l'université du Québec, 232 p.

\section{La psychologie des peuples, la fondation d'un Institut Havrais et A. Miroglio.}


«Abel Miroglio » (1978), Revue de psychologie des peuples, t. 33, n 3.

NICOLLET A. (1999-2000), «Un intellectuel havrais dans le siècle : Abel Miroglio (1895-1978) » dans Cahiers Havrais de Recherche Historique, ${ }^{\circ}$ 58, p. 35-46.

- (1999), Un homme, une ville, une cuvre : Abel Miroglio (1895-1978), Le Havre, Institut Havrais de Sociologie, 127 p. (Repris des Cahiers de Sociologie économique et culturelle, Ethnopsychologie, 30 décembre 1998, p. 9-51).

\section{L'Institut Havrais de psychologie des peuples}

BASTIDE R. (1966), «Y-a-t-il une crise de la psychologie des peuples?» dans Revue de Psychologie des peuples, XXI, $\mathrm{n}^{\circ} 1$.

CALlOT E. (1967), Essais sociologiques, Annecy, Gardet, 277p. (pour une vision de la «psychologie ethnique et culturelle » par un ancien directeur de l'Institut du Havre).

- (1959), «La psychologie des peuples » dans Revue de Psychologie des peuples, t. 14, $\mathrm{n}^{\circ}$ 1, p. 62-71. (Critique du Que-sais-je ? d'A. Miroglio).

CLARET Ph. (1996), «Regards sur l'histoire d'une entreprise intellectuelle : l'Institut havrais et la Revue de psychologie des peuples- Ethnopsychologie (19461982) », Cahiers de Sociologie économique et culturelle-Ethnopsychologie, $\mathrm{n}^{\circ} 26$, décembre, p. 17-36.

- (1996), «Psychologie des peuples ou identités culturelles ? : un regard havrais (1946-1982) », Études Normandes, n 3, p. 29-38.

«Ce que fut l'Institut Havrais de Sociologie économique et de Psychologie des peuples depuis sa fondation (hiver 1937-1938) jusqu'à l'été de 1970 » dans Ethnopsychologie (1978), $\mathrm{n}^{\circ} 3$, p. 241-259.

Institut Havrais de Sociologie économique et de Psychologie des peuples, L'Europe et ses populations, bibliographie, Sup. aux Cahiers de l'Institut, Le Havre, 1993, $41 \mathrm{p}$.

MARANDON S. (1978), «La revue de psychologie des peuples » dans Ethnopsychologie, t. 33, 1978, $\mathrm{n}^{\circ} 3$.

Miroglio A. (1958), «Bilan de vingt années de l'Institut Havrais » dans Revue de Psychologie des Peuples, 113, $\mathrm{n}^{\circ}$ 1, p. 142.

- (1960), «Quelques difficultés majeurs de l'ethnopsychologie » dans Revue de Psychologie des peuples, t.19, $\mathrm{n}^{\circ}$ 3, p. 302-303.

- (1963), «La psychologie des peuples et sa valeur»dans Revue des Sciences Morales et Politiques, 25 février, p. 102-111.

- (1971), La psychologie des peuples, Paris, P.U.F., Que-sais-je ? n ${ }^{\circ} 798,127$ p. (4 éditions 1958, 1962, 1965, 1971), $4^{\mathrm{e}}$ édition.

NICOLLET A. (1995), «André Siegfried à la recherche de la psychologie des normands » dans Cahiers de sociologie économique et culturelle. Ethnopsychologie, 23, juin 1995 , p. 35-45.

\section{L'histoire de la sociologie}

BORLANDI M. et MUCCHIELLI L. (1995), (S. Dir.), La sociologie et sa méthode. Les règles de Durkheim un siècle après, Paris, L'Harmattan, 416 p.

ROBIC M.-Cl., «Entre sociologie et géographie : la morphologie sociale selon Durkheim », p. 101-136. 
1 N. BANCEL, P. BLANCHARD, F. VERGES, La République coloniale, Paris, Pluriel, 2003, 174 p.

2 L. MUCCHIELLI (1998), «L'obstacle de la psychologie des peuples » et «Le modèle de l'enracinement dans le sol naturel » dans La découverte du social : naissance de la sociologie en France, Paris, La Découverte, 1998, p. 319-321 et p. 384-387.

3 Présidée par Charcot, cette société regroupa, entre autres, Paul et Pierre Janet, Théodule Ribot, Charles Richet, Charles Féré, Émile Gley, Léon Marillier mais aussi Sully-Prudhomme, Taine et Gabriel Tarde.

4 Sur A. Fouillée et la «psychologie des Peuples » voir G. NOIRIEL, Les origines républicaines de Vichy, Paris, Hachette, 1999, 335 p, p. 245-247. Sur Gustave Le Bon voir Y. THIEC, «Gustave Le Bon, prophète de l'irrationalisme » dans Revue Française de Sociologie, 1981, vol. 22, n 3, p. 409-428 et Gustave Le Bon, la psychologie des foules, la fondation de la psychologie collective et sa propagation dans les sciences sociales à la fin du XIX ${ }^{e}$ siècle, Institut universitaire européen, thèse de doctorat, 1982.

5 Pour cette partie nous nous appuyons essentiellement sur le mémoire de Le Quintrec G. (1991 et 1989), «Alfred Fouillée et la psychologie des peuples », Cahiers de Sociologie, Economique et Culturelle, $\mathrm{n}^{\circ} 15$, juin, p. 35-60. (article issu d'un mémoire de D.E.A., Dir. P. Nora, E.H.E.S.S, 1989). Voir aussi Claret Ph. (1999), CLARET Ph. (1999), «Psychologie collective, psychologie des peuples et Science politique en France depuis 1870 : histoire d'un rendez-vous manqué » dans La psychologie des peuples et ses dérives, Op. Cit., p. 63-80 et Rosanvallon P. (1998), Le peuple introuvable. Histoire de la représentation démocratique en France, Paris, Gallimard. (essentiellement le chapitre «Sociologie et démocratie », p. 130-175.)

6 Ozouf J. et Ozouf M. (1984), « Le tour de France par deux enfants, Le petit livre rouge de la République » dans Les lieux de mémoire (S. Dir. P. Nora), t. 1, La République, Paris, Gallimard, p. 291-321.

7 Sur l'anthropo-sociologie voir Ammon O. (1988), « Histoire d'une idée : l'anthropo-sociologie » dans Revue internationale de Sociologie, $6^{\mathrm{e}}$ année, $\mathrm{n}^{\circ} 3$.

8 Sur Vacher de Lapouge voir Thuillier (G.) (1977), « Un anarchiste positiviste : Georges Vacher de Lapouge » dans Guiral P. et Témine E., L'idée de race dans la pensée politique française contemporaine, Paris, Ed. du C.N.R.S., p. 48-65.

9 Cité dans G. NOIRIEL, op. cit., p. 245-246.

$10 \quad$ Nye Robert A. (1975), op. cit.

11 Liauzu Cl. (1992), op. cit. 
12 Voir L'Année Psychologique (années 1922 et 1928) et Liauzu Cl. (1992), op. cit., p. 112-113.

13 Nous écartons ici la question de l'eugénisme qui eut pourtant de réels soutiens en France (et qui fut un aspect important du contexte de l'entre deuxguerres) notamment avec la création d'une Société française d'eugénique, fin 1912, sur le modèle anglais et sous le patronage de Léon Bourgeois (alors ministre du Travail et de la Prévoyance sociale) et de Charles Richet (Prix Nobel de médecine en 1913) et qui eut des prolongements encore plus ténébreux dans les travaux d'Alexis Carrel (Prix Nobel de médecine en 1912).

14 Barrows S. (1990), op. cit. et Moscovici S. (1985), op. cit.

15 Voir Noiriel G. (1999), op. cit., p. 231.

16 Rosanvallon P. (1998), op. cit. ; Balibar E. et Wallerstein E. (1997), op. cit. ; Noiriel G. (2001), op. cit. et Arendt H. (1968), op. cit.

17 G. Noiriel place bien les interrogations intellectuelles d'A. Siegfried à la suite de Maurice Barrès, Vidal de la Blache et Le Bon. Noiriel G. (1988), op. cit., p. 34-35.

18 Claret Ph. (1995), op. cit. ; Bonnefous E. (1975), op. cit. ; Célébration du centenaire de la naissance d'A. Siegfried (1975), op. cit. ; Comité pour le centenaire de la naissance d'André Siegfried (1975), op. cit. ; L'œuvre scientifique d'André Siegfried (1977), op. cit. ; Pommier J. (1961), op. cit.

19 Voir Sternhell Z. (2000), op. cit. ; Birnbaum P. (1993) mais aussi Rousso H. (1990), p. 282-283, p. 287-288 et p. 353.

20 Voir sur ce point voir Sternhell Z. (2000), op. cit. ; Birnbaum P. (1993) ; Milza P. (1995 et 1987), op. cit.

21 Voir Andler Ch., (1932), op. cit., p. 50-56. et p. 35. Sur Charles Andler, le Pangermanisme et le racisme «néo-darwinien » voir Nicolet Cl. (2006), op. cit., p. 239 et p. 274-275.

22 Voir Lequin Y. (2006), op. cit. p.346.

23 Voir Birnbaum P. (1993) et Noiriel G. (1999), op. cit., p. 260-261.

24 Sur Jules Soury et Maurice Barrès voir surtout Sternhell Z. (1977), op. cit. et du même (1997), « Le darwinisme social et la découverte de l'inconscient », p. 179-197. Voir aussi Gauchet M. (1992), «La pensée, le langage et la foule » op. cit., p. 105-126.

25 Voir Sternhell Z. (2000), op. cit. ; Birnbaum P. (1993), op. cit. et du même (1992), p. 442. Un antisémitisme « larvé » que l'on put à nouveau percevoir dans les analyses d'André Siegfried concernant l'« entourage » de Pierre Mendès France sous 
la IV ${ }^{\mathrm{e}}$ République, par exemple, mais aussi plus largement dans ses études (qui ont fait longtemps succès en sciences politiques sur le Régime de Vichy : à savoir la thèse distinguant fondamentalement un « Vichy de Pétain » d'un « Vichy de Laval » diffusée par André Siegfried puis vulgarisée par Robert Aron. Une « histoire officielle » longtemps non discutée par les historiens français jusqu'aux travaux de l'historien américain Robert Paxton, voir Noiriel G. (1999), op. cit., p. 254-261; Azéma J.-P. et Wieviorka O., op. cit., p.346).

26 Pour une critique vigoureuse de l'oeuvre scientifique d'A. Siegfried voir Sternhell Z. (2000), op. cit., p. 23-26, p. 28-37 et p. .39-43.

27 Garrigou (A.), (1995), op. cit. «L'ouest politique 75 ans après Siegfried » (1987), op. cit. et Milza P. (1995), op. cit.

28 D'ailleurs n'est-il pas cité en exergue par l'historien Philippe Ariès dont on connaît aussi, dans sa formation initiale, le poids de l'Action Française dont il ne s'est jamais caché ? Voir Noiriel G. (1999), op. cit., p.254.

29 Sur Ratzel voir «La géographie politique de Ratzel » dans Moreau de Farges Ph. (2005), op. cit., p. 83-88. ; Voir aussi Mucchelli L. et Robic M.-Cl. (1995), op. cit.

30 Voir Sternhell Z., op. cit., p. 29-30. et Claret Ph. (1993, 1998 et 2001), op. cit.

31 Siegfried A. (1913), op. cit., p.39.

32 Miroglio A. (1971), op. cit., p.30.

33 Edward Gibbon Wakefield (1796-1862) était le fils d'Edouard Wakefield, statisticien. Il étudia d'abord en Angleterre où il était né puis en Écosse. Il fut emprisonné de 1827 à 1830 à la suite de l'enlèvement d'une fille d'un fabricant de soie du Cheshire. À cette occasion il fit paraître à Londres un ouvrage dans lequel il exposait ses théories concernant la colonisation. Il fut surtout l'inspirateur de la South Australian Association puis de la New Zealand Association. Par la suite il dirigea la New Zealand Colonization Company, puis devint agent de la North American Colonial Association of Ireland avant de devenir député du Beauharnois canadien en 1842 . Il se retira en Nouvelle-Zélande en 1844. Voir Siegfried A. (1904), op . cit. ainsi que le site de l'Assemblée Nationale du Québec (http://www.assnat.qc. ca).

34 Miroglio A. (1978), op. cit. et Nicollet A. (1989), op. cit. Sur ce point voir aussi les 4 volumes sur Le Pangermanisme de Charles Andler « la plus complète anthologie des sources du nazisme qu'on puisse consulter » selon Claude Nicolet (2006), op. cit., « Nos ancêtres les Gaulois », p. 239-240.

Claval P. (1989), op. cit. 
36 Vidal de la Blache P. (1914), op. cit. Sur Vidal de la Blache voir Moreau Defarges Ph. (2005), op. cit., p. 119-123 (qui ne mentionne curieusement jamais A. Siegfried).

37 Berr H. (1903), op. cit. ; Lorquet P. (1901), op. cit. ; Poncin P. (1900), op. cit. . Voir aussi Mucchielli L. (1997), op. cit. et Zeldin Th. (2002), op. cit., vol. 2, p. 53-55. ; vol. 4, p. 22-39, p.41 et p.43.

38 Pour une analyse de «l'anti-américanisme » français en lien avec l'ouvrage d'André Siegfried, qui, contrairement à l'opinion répandue à cette époque, est tout à fait admiratif du modèle « anglo-saxon » tout en montrant ses faiblesses, voir Winock M. (2004), op. cit., p. 52-53.

39 Voir Noiriel G. (1999), op. cit., « Le tempérament national », p. 234-237. et p. 277 (l'article 6 de la loi de 1927 qui permet la « naturalisation » après enquête). Ces réflexions seront reprises par André Siegfried après la Libération dans ses cours de la rue Saint-Guillaume à l'IEP de Paris de 1947 à 1957.

40 Favre P. (1989), op. cit. (notamment la $4^{\mathrm{e}}$ partie, p. 233-306).

41 Sternhell Z. (2000), op. cit.

42 Noiriel (G.) (1999), Les origines républicaines de Vichy, Paris, Hachette, p. 254-261. et p.323. Les allusions antisémites et racistes d'André Siegfried sur les tensions sociales et raciales aux Etats-Unis sont particulièrement nettes. Gérard Noiriel posait ainsi la question : «Comment un intellectuel, sincèrement républicain par ailleurs, a-t-il pu reprendre ainsi à son compte de tels stéréotypes racistes et antisémites ? » Voir aussi G. NOIRIEL (2007), Immigration, antisémitisme et racisme en France (XIXe-XXe siècle). Discours publics, humiliations privées, Paris, Fayard., p.512. et p.677-677.

43 Siegfried A. (1927) titre de la traduction anglaise.

44 Lavisse E., Essai sur l'Allemagne impériale, Paris, 1888 cité par Nicolet Cl., op. cit., p. 280.

45 Favre P. (1981), op. cit.

46 Sur cet aspect voir Chambelland C. (1998), op. cit. et Ardaillou P. (1999), op. cit.

47 Ardaillou P. (1992), op. cit. ; Legoy J. (1987), op. cit. ; Merlin R. (1923), op. cit. ; Siegfried A. (1946) op. cit. ; Vallée T. (1910), op. cit. et « Jules Siegfried aux États-Unis » (1955), Livres de France, $\mathrm{n}^{\circ} 2$, février, op. cit. (sources).

$48 \quad$ Sur cet aspect voir Legoy J. (1982, 1984 et 1986).

49 Zeldin Th. (2002), op. cit., vol.1, p. 112-113. 
50 Bibliothèque municipale du Havre, voir dossier $92 \mathrm{~N}$ : « Jules Siegfried aux États-Unis » (1955), Livres de France, $\mathrm{n}^{\circ} 2$, février.

51 Sur ce point voir Girardet R. (1972), op. cit., p. 113 et p. 115.

52 Sur la sociologie économique et sa diffusion voir Gislain J.-J. et Steiner Ph. (1995), La sociologie économique (1890-1920), Paris, P.U.F., 235 p.

et Steiner Ph. (1999), La Sociologie économique, Paris, La découverte, coll. Repères, p. 3-5. Voir aussi Encrevé A. et Richard D. (1979), op. cit. ; Garrisson-Estèbe J. (1980), op. cit. ; Weber M. (1991), op. cit. et Nicollet A. (1996), op. cit.

53 Letourneau Ph. (1884, 1895 et 1901), op. cit. (sources). Voir aussi Noiriel G. (1999), op. cit., p. 230-231. et Borlandi M. et Mucchielli L. (1995), op. cit., p. 45-47.

$54 \quad$ Voir Bancel N., Blanchard P., Vergès F., « Une métaphore de la République coloniale, l'Exposition de $1931 »$, p. 110-115.

55 Miroglio A. (Sans date), op. cit.

$56 \quad$ Miroglio A. (1967), op. cit.

${ }^{57}$ Hardy G. (1939), op. cit. Georges Hardy était en fait l'instituteur Gabriel Giroud, le gendre de Paul Robin, théoricien du néomalthusianisme, fondateur de la Revue Régénération, fin connaisseur du mouvement eugéniste américain et des idées d'Alexis Carrel, avant la $1^{\text {ère }}$ guerre mondiale. Voir Drouard A. «Alexis Carrel et l'eugénisme » dans Des sciences contre l'homme (1993), vol.II, «Au nom du bien », p.33 (note de bas de page $\left.\mathrm{n}^{\circ} 4\right)$.

$58 \quad$ Claval P. (1989), op. cit. et Wylie (L.) (1977), op. cit.

59 Miroglio A. (1945), op. cit. Sur la «géographie politique » voir Sanguin A. (1976 et 1985), op. cit.

60 Zeldin Th. (2002), op. cit., vol. 4., p. 22. Voir aussi Goguel F. (1959), op. cit.

61 Miroglio A. (1971), op. cit., p. 71-72.

62 Ethno-psychologie (1978), op . cit. Pour le contexte colonial et économique voir Girault R. et Frank R. (1997 et 1998), op. cit. ; Marseille J. (1986), op. cit. Voir aussi Malon Cl. (1997), op. cit. et Miroglio A. (1958), op. cit.

63 Callot E., (1967), op. cit. (pour une vision de la « psychologie ethnique et culturelle » par un ancien directeur de l'Institut du Havre). et du même (1959), op. cit.

64 Pour le contexte voir Lautman J., « Chronique de la sociologie française après 1950 » dans Boudon R., Bourricaud F., Girard A. (1981), op. cit., p. 269-284.

65 Elle avait accueilli notamment au cours de ses conférences (et pour des contributions) Leroy-Beaulieu, Lévy-Bruhl et André Siegfried. La bibliothèque de 
cette société rassemblait environ 4191 ouvrages proposant une documentation aux hommes d'affaires. Voir aussi Lejeune D. (1993), op. cit.

66

Miroglio A. (1960), op. cit.

67 Voir sur ce point Bancel N., Blanchard P., Vergès F. (2003), op. cit.

(notamment les chapitres «Droits et devoirs de la mission civilisatrice », p. 67-88;

«Race et Nation républicaine », p. 89-128.)

68 Voir aux Archives départementales la Seine-Maritime la Série 4M574 :

Associations, enseignement et propagande coloniale.

69 Voir les Programmes et statuts aux archives départementales de la SeineMaritime.

70 Pour le contexte national voir Blanchard P. et Lemaire S. (2004), op. cit. voir aussi Manceron G. (2003), op. cit.

Miroglio A. (1971), op. cit.

72 Zeldin Th. (2002), op. cit., vol. 5, p. 437-441. Sur le contexte et l'essor de ces préoccupations voir Sauvy A. et Ledermann S. (Juillet-Septembre 1946), « La guerre biologique (1933-1945) : population de l'Allemagne et des pays voisins » dans Population, $\mathrm{n}^{\circ}$ 3, p. 471-488. Pour les liens avec la démographie voir Lebras H. (2006), op. cit., p. 42-43.

73 Voir Noiriel (G.) (1999), «La Fondation pour l'étude des problèmes humains », p. 217-222. et Gillon J.-J. « La Fondation française pour l'étude des problèmes humains » dans Boudon R., Bourricaud F., Girard A. (1981), op. cit., p. 257-268. Devenue Fondation de l'Etat Français le 17 novembre 1941 avec l'objectif de « sauvegarder, améliorer et développer la population française », ces activités regroupèrent « paradoxalement » des personnalités aussi différentes que Françoise Dolto, Jean Stoetzel, Louis Chevalier et Pierre Naville. Sur l'Institut Rockefeller et «L'instrumentalisation des sciences sociales » en France voir, avec précaution, sur le site internet Voltaire édition internationale, l'article de Chavaux B. (7 juillet 2004), «Guerre froide. EHESS : les sciences sociales françaises sous perfusion de la CIA », p. 1-3. (www.voltairenet.org).

$74 \quad$ Sur Jean Coutrot voir les Fonds des Archives nationales et le centre d'histoire de Science-Po : Archives d'histoire contemporaine : http://centre-histoireSciences-po-fr/ archives/ fonds/Jean-Coutrot-html (Notice bibliographique et Fonds du Centre d'étude des problèmes humains 1838-1839 Cotes : 468AP/21 à 468AP/25).

75 Sur le principal dirigeant de l'Institut voir Revue de psychologie des peuples (1978), op. cit. ; Nicollet A. (1999-2000), op. cit. Voir aussi Nicollet A. (1999), op. cit. 
77 Claret Ph. (1996), op. cit. et Miroglio A. (1963), op. cit.

78 Bastide R. (1966), op. cit.

79 Malon Cl. (1997), op. cit.

80 D'après les Archives conservées à la Bibliothèque Municipale du Havre et Malon (Cl.) (1997), op. cit. Pour le contexte voir aussi Ageron Ch. R. (1991), op. cit.

81 D'après les statuts, programmes, imprimés listes des membres conservés aux Archives départementales de la Seine-Maritime et l'assemblée générale du 2710-1938 (Cote : 4M575) et Malon Cl. (1997), op. cit.

82 Hardy G. (1947), op. cit.

83 Teillac J. (1954), op. cit.

84 Pour un aperçu des travaux de G. Balandier voir du même « Ethnologie, anthropologie, sociologie » dans Boudon R., Bourricaud F., Girard A. (1981), op. cit., p. 71-78.

85 Pour le contexte continental et international voir Vaïsse M. (1991), op. cit. ainsi que Girault F., Franck R. Thobie J. (2005), op. cit.

86 Revue de Psychologie des Peuples, 1963, n 4, p. 496-503.

87 Miroglio A. (1971), op. cit., p. 74-75.

88 Miroglio A. (1978), op. cit.

89 Voir Duroselle J.-B. (1990), p. 569.

90 Pour un aperçu des études de Jean Stoetzel et de ces disciples sur la «psychologie des peuples » en rapport avec la «psychologie sociale » on pourra consulter les articles de Kon I., « Le concept de personnalité », Klineberg, « Jean Stoetzel et l'étude scientifique du caractère national », et Dogan M., « La transmission « héréditaires » des tendances politiques » dans Boudon R., Bourricaud F., Girard A. (1981), op. cit., p. 30-41, p. 42-48. et p. 216-228.

91 Marandon S., (1978), op. cit.

92 Cuin Ch.-H. et Gresle F. (2002), op. cit., t. 2, p. 57-60.

93 Birnbaum P. (1993), op. cit. 